\title{
On the automorphisms of a graph product of abelian groups
}

\author{
Mauricio Gutierrez, Adam Piggott and Kim Ruane
}

\begin{abstract}
We study the automorphisms of a graph product of finitely generated abelian groups $W$. More precisely, we study a natural subgroup Aut ${ }^{*} W$ of Aut $W$, with Aut ${ }^{*} W=$ Aut $W$ whenever vertex groups are finite and in a number of other cases. We prove a number of structure results, including a semi-direct product decomposition Aut ${ }^{*} W=\left(\operatorname{Inn} W \rtimes \mathrm{Out}^{0} W\right) \rtimes$ Aut ${ }^{1} W$. We also give a number of applications, some of which are geometric in nature.
\end{abstract}

Mathematics Subject Classification (2010). 20F28, 20 F65.

Keywords. Automorphism groups, graph products of groups, right-angled Coxeter groups, right-angled Artin groups.

\section{Introduction}

The graph product of groups construction was first defined by Green [13]. It interpolates between the free product construction, in the case that $\Gamma$ is a discrete graph, and the direct product construction, in the case that $\Gamma$ is a complete graph. The class of graph products of finitely generated abelian groups contains a number of important subclasses that are often treated separately, including finitely generated abelian groups, graph products of primary cyclic groups, right-angled Coxeter groups and right-angled Artin groups. In the present article we pursue a unified treatment of the automorphisms of such groups. Our methods are combinatorial. Our results have a number of applications which are geometric in nature.

The class of graph products of finitely generated abelian groups is identical to the class of graph products of directly indecomposable cyclic groups, and it is more convenient to consider the latter. Groups in this class are in one-to-one correspondence with the labeled-graph isomorphism classes of non-trivial finite simplicial graphs in which vertices are labeled by either a prime power or infinity [16]. Such a labeled graph $\Gamma$ determines a canonical group presentation of a group $W=W(\Gamma)$, where the vertices of $\Gamma$ are in one-to-one correspondence with the generators of $W$, the label on each vertex specifies the order of the corresponding generator, and adjacency in the graph determines when generators commute. In the case that each vertex of $\Gamma$ is 
labeled by a prime power, we say that $W$ is a graph product of primary cyclic groups. Note that a right-angled Coxeter group is a graph product of primary cyclic groups.

A subgroup of $W$ which is generated by the vertices in a maximal complete subgraph $\Delta \subset \Gamma$ is called a maximal complete subgroup. Such subgroups are important to our study because if $W$ is a graph product of primary cyclic groups, then the maximal complete subgroups are a set of representatives for the conjugacy classes of maximal finite subgroups of $W$ [13], Lemma 4.5, and each automorphism of $W$ maps each maximal complete subgroup to a conjugate of some maximal complete subgroup. This is not true in an arbitrary graph product of directly indecomposable cyclic groups, but we may pretend that it is by restricting our attention to a natural subgroup of Aut $W$. By doing so we are able to extend the application of ideas which work for the automorphisms of graph products of primary cyclic groups.

Definition 1.1. Write Aut* $W$ for the subgroup of Aut $W$ consisting of those automorphisms which map each maximal complete subgroup to a conjugate of a maximal complete subgroup.

While studying Aut $W$ is not the same as studying Aut $W$ in all cases, we note that the equality Aut ${ }^{*} W=$ Aut $W$ holds in the case that $W$ is a graph product of primary cyclic groups, and in many other interesting cases (see Lemma 2.8 below). In particular, our study includes the study of the full automorphism groups of rightangled Coxeter groups.

We are now ready to report the main results of the present article. They concern the structure of Aut* $W$ and shall make reference to the subgroups and quotients of Aut $W$ defined in Table $1 .{ }^{1}$ It is natural to expect that a unified treatment of a class of groups will give results which generalize results known for subclasses. While some of our results and applications do this, each of our main theorems, and a number of their applications, offers something new even in the case that $W$ is a right-angled Coxeter group.

In Section 3 we exhibit an iterated semidirect product decomposition of Aut* $W$.

Theorem 1.2. Aut ${ }^{*} W=\underbrace{\left(\operatorname{Inn} W \rtimes \operatorname{Out}^{0} W\right)}_{\operatorname{Aut}^{0} W} \rtimes \operatorname{Aut}^{1} W$.

Tits [30] proved that Aut $W=\operatorname{Aut}^{0} W \rtimes \operatorname{Aut}^{1} W$ in the special case that $W$ is a right-angled Coxeter group. That $\operatorname{Aut}^{0} W=\operatorname{Inn} W \rtimes \operatorname{Out}^{0} W$ was recently established in the special case that $W$ is a right-angled Coxeter group satisfying certain graph restrictions [14], and is also apparent in the special case considered in [9]. We use different methods to establish our more general result. In particular, the

\footnotetext{
${ }^{1}$ In writing Aut $^{0} W$ for the subgroup of 'conjugating automorphisms', we follow Tits [30]. Mühlherr [28] writes Spe $(W)$ for the same subgroup. Charney, Crisp and Vogtmann [6] use the notation $\operatorname{Aut}^{0} W$ and Out $^{0} W$ for different subgroups of the automorphism group of a right-angled Artin group than described here.
} 
Table 1. Subgroups and quotients of Aut $W$.

\begin{tabular}{|l|l|}
\hline Group & Description \\
\hline Aut* $W$ & $\begin{array}{l}\text { Those automorphisms of } W \text { which map each maximal complete } \\
\text { subgroup to a conjugate of a maximal complete subgroup }\end{array}$ \\
\hline Aut $^{1} W$ & $\begin{array}{l}\text { Those automorphisms of } W \text { which map each maximal complete } \\
\text { subgroup to a maximal complete subgroup }\end{array}$ \\
\hline Aut $^{0} W$ & $\begin{array}{l}\text { Those automorphisms of } W \text { which map each vertex } v_{i} \in V \\
\text { to a conjugate of itself }\end{array}$ \\
\hline Inn $W$ & The inner automorphisms of $W$ \\
\hline Out $W$ & The quotient Aut $W /$ Inn $W$ \\
\hline Out $^{*} W$ & The quotient Aut ${ }^{*} W /$ Inn $W$ \\
\hline Out $^{0} W$ & The quotient Aut $^{0} W / \operatorname{Inn} W$ \\
\hline
\end{tabular}

new technical tool we use to prove Theorem 1.2 is the Restricted Alphabet Rewriting Lemma of Subsection 3.2. It is a combinatorial lemma which establishes that certain subgroups of $\mathrm{Aut}^{0} W$ are convex inside Aut $^{0} W$ in a particularly strong way: by looking at the images of generators under an automorphism $\phi \in \operatorname{Aut}^{0} W$, one may immediately identify certain letters in a word for $\phi$ as unnecessary.

In a mild abuse of notation, we shall identify Out $^{0} W$, which is defined as a quotient group, with a particular subgroup of $\mathrm{Aut}^{0} \mathrm{~W}$, as suggested by Theorem 1.2.

Theorem 1.2 has application to the theory of group extensions, as discussed in Section 3.4. It also serves to identify the subgroup $\mathrm{Out}^{0} W$ as the key to understanding Aut ${ }^{*} W$ and Out* $W$, since the subgroups Inn $W$ and Aut ${ }^{1} W$ are, at least in some sense, well-understood (see Lemma 2.7 and Remark 2.10). Our method of proof suggests a convenient set of generators $\mathcal{P}^{0}$ for Out ${ }^{0} W$ (see Definition 3.16).

In Section 4 we investigate the structure and cardinality of $\mathrm{Out}^{0} \mathrm{~W}$. In particular, we identify a simple type of subgraph whose presence or absence determines much about the automorphism groups of $W$. In the following, we write $L_{i}$ for the link of a vertex $v_{i}$.

Definition 1.3 (SIL). We say that $\Gamma$ contains a separating intersection of links (SIL) if there exist vertices $v_{i}$ and $v_{j}$ such that the following conditions hold:

(1) $d\left(v_{i}, v_{j}\right) \geq 2$

(2) there exists a connected component $R$ of $\Gamma \backslash\left(L_{i} \cap L_{j}\right)$ such that $v_{i}, v_{j} \notin R$. 
Theorem 1.4. If $W$ is a graph product of directly indecomposable cyclic groups, then the following are equivalent:

(1) $\mathrm{Out}^{0} W$ is an abelian group;

(2) $\Gamma$ does not contain a SIL.

If $W$ is a graph product of primary cyclic groups, then the following is also equivalent to properties (1) and (2) above:

(3) Out $W$ is finite.

Levitt [23] showed that, for a one-ended word hyperbolic group $G$, Out $(G)$ is infinite if and only if $G$ splits over a virtually cyclic subgroup with infinite center, either as an arbitrary HNN extension or as an amalgam of groups with finite center. It follows from our results that such splittings are not possible in the case that $W$ is a graph product of primary cyclic groups (see Corollary 4.8). This illustrates the utility of a result like Theorem 1.4: once one has identified a property of a labeled graph which characterizes a property of the associated graph product of groups, then one may examine the combined effect of the existence (or absence) of that graph property and other graph properties known to characterize group properties. We follow this program to examine the combined effect of the existence (or absence) of an SIL and certain graph properties which are known to determine geometric properties of $W$. The properties we consider include the isolated flats property (Corollary 4.11) and whether or not $W$ can act on a CAT(0) space with locally-connected visual boundary (Corollary 4.12). We also characterize when Aut $W$ is word hyperbolic (Corollary 4.14).

In Section 5 we investigate the special case that $\Gamma$ is a tree, in which our study of Out $^{0} W$ can proceed much further. We prove the following.

Theorem 1.5. If $W$ is a graph product of directly indecomposable cyclic groups and $\Gamma$ is a tree with vertices $v_{1}, v_{2}, \ldots, v_{N}$, then

$$
\operatorname{Out}^{0} W \cong \mathrm{Ab} \times\left(\prod_{i=1}^{N} \operatorname{Out}^{0} W\left(L_{i}\right)\right)
$$

for a finitely generated abelian group $\mathrm{Ab}$ as described in Remark 5.6. In particular:

(1) if $W$ is a right-angled Artin group, then $\mathrm{Ab}$ is a free abelian group;

(2) if $W$ is a graph product of primary cyclic groups, then $\mathrm{Ab}$ is a finite abelian group.

From this, we are able to determine a finite presentation for Aut* $W$ (Remark 5.6), to calculate the virtual cohomological dimension of Out $W$ in the case that $W$ is a graph product of primary cyclic groups (Corollary 5.7) and to prove the existence of regular languages of normal forms for $\mathrm{Out}^{0} W$ and $\mathrm{Aut}^{0} W$ in the case that $W$ is a right-angled Artin group (Corollary 5.8). 
Because we are attempting a unified treatment of a class of groups which has a number of much studied subclasses, our results overlap with those of many authors. We mention that Laurence [21] and Mühlherr [28] independently determined finite presentations for $\mathrm{Aut}^{0} \mathrm{~W}$ in the case that $W$ is a right-angled Coxeter group. Castella [5] determined a finite presentation of $\mathrm{Aut}^{1} \mathrm{~W}$ for a certain subclass of right-angled Coxeter groups. Proving a conjecture of Servatius [29], Laurence [22] determined a finite generating set for Aut $W$ in the case that $W$ is a right-angled Artin group - a generating set for Aut* $W$ can be deduced from this list - and Day [10] determined a finite presentation for Aut $W$ in this case. In a recent series of papers, Bux, Charney, Crisp and Vogtmann have developed a new topological approach to the study of Out $W$ in the case that $W$ is a right-angled Artin group [6], [7], [4]. To the best of the authors' knowledge, Laurence's unpublished Ph.D. thesis [21] is the only previous work to consider the automorphisms of graph products of abelian groups in a unified way.

\section{Preliminaries}

In this section we establish notation, remind the reader of some fundamental results concerning graph products of groups and justify several statements made in the introduction. Our notation is tailored specifically to the class of graph products of directly indecomposable cyclic groups, and we have stated the results for this class of groups only. The results appear, in more general form, in Elisabeth Green's Ph.D. thesis [13] and Michael Laurence's Ph.D. thesis [21], proved by different methods.

A non-trivial finite simplicial graph $\Gamma=\Gamma(V, E)$ is a pair consisting of a nonempty finite set $V=\left\{v_{1}, v_{2}, \ldots, v_{N}\right\}$ (the vertices) and a set $E$ (the edges) of unordered pairs from $V$. We say that vertices $v_{i}, v_{j}$ are adjacent if $\left\{v_{i}, v_{j}\right\} \in E$. We consider $\Gamma$ to be a metric object in the usual way, with $d_{\Gamma}$ denoting the distance function. An order map (on $\Gamma$ ) is a function

$$
\boldsymbol{m}:\{1,2, \ldots, N\} \rightarrow\left\{p^{\alpha} \mid p \text { prime and } \alpha \in \mathbb{N}\right\} \cup\{\infty\} .
$$

A pair $(\Gamma, \boldsymbol{m})$ is called a labeled graph and determines a group $W(\Gamma, \boldsymbol{m})$ with the following presentation (by convention, the relation $v_{i}^{\infty}$ is the trivial relation):

$$
\left\langle V \mid v_{i}^{\boldsymbol{m}(i)}, v_{j} v_{k} v_{j}^{-1} v_{k}^{-1} \quad\left(1 \leq i, j, k \leq N, j<k, d_{\Gamma}\left(v_{j}, v_{k}\right)=1\right)\right\rangle .
$$

We say that $W(\Gamma, \boldsymbol{m})$ is a graph product of directly indecomposable cyclic groups. Following an established convention, we do not distinguish between a vertex of $\Gamma$ and the corresponding generator of $W(\Gamma, \boldsymbol{m})$.

Restricting $\Gamma$ or $\boldsymbol{m}$, or both, gives the important subclasses mentioned in the opening paragraph of the introduction, namely: abelian groups ( $\Gamma$ a complete graph); graph products of primary cyclic groups $(\boldsymbol{m}(i)<\infty$ for each $i)$; right-angled Coxeter groups $(\boldsymbol{m}(i)=2$ for each $i)$; and right-angled Artin groups $(\boldsymbol{m}(i)=\infty$ for each $i)$. 
The class of graph products of directly indecomposable cyclic groups is identical to the class of graph products of finitely generated abelian groups for the following reason: if $G$ is group and $G$ is isomorphic to a graph product of finitely generated abelian groups, then there exists a unique isomorphism class of labeled graphs $(\Gamma, \boldsymbol{m})$ such that $G \cong W(\Gamma, \boldsymbol{m})$ [16]. Empowered by this fact, we usually omit mention of $\Gamma$ and $\boldsymbol{m}$ from the notation, writing $W:=W(\Gamma, \boldsymbol{m})$.

By a subgraph of $\Gamma$ we shall always mean a full subgraph. Thus a subgraph $\Delta=\left(V_{\Delta}, E_{\Delta}\right)$ is determined by a subset $V_{\Delta} \subseteq V$ and the rule $E_{\Delta}=\left\{\left\{v_{i}, v_{j}\right\} \in\right.$ $\left.E \mid v_{i}, v_{j} \in V_{\Delta}\right\}$.

Definition 2.1. Let $1 \leq i_{1}, \ldots, i_{k} \leq N$ be such that $i_{j} \neq i_{j+1}$ and let $\alpha_{1}, \ldots, \alpha_{k}$ be non-zero integers. Each $v_{i_{j}}^{\alpha_{j}}$ is a syllable of the word $v_{i_{1}}^{\alpha_{1}} v_{i_{2}}^{\alpha_{2}} \ldots v_{i_{k}}^{\alpha_{k}}$, and we say that the word is reduced if there is no word with fewer syllables which spells the same element of $W$. We say that consecutive syllables $v_{i_{j}}^{\alpha_{j}}, v_{i_{j+1}}^{\alpha_{j+1}}$ are adjacent if $v_{i_{j}}$ and $v_{i_{j+1}}$ are.

Lemma 2.2 (The deletion condition). Let $1 \leq i_{1}, \ldots, i_{k} \leq N$ be such that $i_{j} \neq i_{j+1}$ and let $\alpha_{1}, \ldots, \alpha_{k}$ be non-zero integers. If the word $v_{i_{1}}^{\alpha_{1}} v_{i_{2}}^{\alpha_{2}} \ldots v_{i_{k}}^{\alpha_{k}}$ is not reduced, then there exist $p, q$ such that $1 \leq p<q \leq k, v_{i_{p}}=v_{i_{q}}$ and $v_{i_{p}}$ is adjacent to each vertex $v_{i_{p+1}}, v_{i_{p+2}}, \ldots, v_{i_{q-1}}$.

Lemma 2.3 (Normal form). Let $1 \leq i_{1}, \ldots, i_{k}, j_{1}, \ldots, j_{k} \leq N$ and $\alpha_{1}, \ldots, \alpha_{k}$, $\beta_{1}, \beta_{2}, \ldots, \beta_{k} \in \mathbb{Z} \backslash\{0\}$. If $v_{i_{1}}^{\alpha_{1}} v_{i_{2}}^{\alpha_{2}} \ldots v_{i_{k}}^{\alpha_{k}}$ and $w_{j_{1}}^{\beta_{1}} w_{j_{2}}^{\beta_{2}} \ldots w_{j_{k}}^{\beta_{k}}$ are reduced words which spell the same element of $W$, then the first word may be transformed into the second by repeatedly swapping the order of adjacent syllables.

The centralizer of a vertex is easily understood.

Lemma 2.4. For each $1 \leq j \leq N$, the centralizer of $v_{j}$ in $W$ is the special subgroup generated by $S_{j}$.

For a full subgraph $\Delta$ of $\Gamma$, we write $W(\Delta)$ for the subgroup (known as a special subgroup) of $W$ generated by the vertices in $\Delta$. We write $\operatorname{MCS}(\Gamma)$ for the set of maximal complete subgraphs (or cliques) of $\Gamma$. As mentioned in the introduction, the subgroups $W(\Delta), \Delta \in \operatorname{MCS}(\Gamma)$, are called the maximal complete subgroups of $W$. The following three results witness the importance of special subgroups to the study of $W$.

Lemma 2.5. Let $\Delta$ be a subgraph of $\Gamma$. The map $V_{\Gamma} \rightarrow W(\Delta)$ defined by

$$
v \mapsto \begin{cases}v & \text { if } v \in V_{\Delta}, \\ 1 & \text { otherwise, }\end{cases}
$$

extends to a retraction homomorphism $p_{\Delta}: W(\Gamma) \rightarrow W(\Delta)$. Further, the natural map $W(\Delta) \rightarrow W(\Gamma)$ is an embedding. 
Lemma 2.6. A special subgroup $W(\Delta)$ has finite order if and only if $\Delta$ is a complete graph and each vertex of $\Delta$ has finite order. Further, if a subgroup $H$ of $W$ has finite order, then $H$ is contained in some conjugate of a special subgroup of finite order.

Lemma 2.7. The center of $W$ is the special subgroup generated by the vertices $\left\{v_{i} \in V \mid d_{\Gamma}\left(v_{i}, v_{j}\right) \leq 1\right.$ for each $\left.1 \leq j \leq N\right\}$. Further, the center of $W$ is finite in the case that $W$ is a graph product of primary cyclic groups.

It follows that Inn $W$ is isomorphic to the special subgroup generated by the vertices $\left\{v_{i} \in V \mid d_{\Gamma}\left(v_{i}, v_{j}\right)>1\right.$ for some $\left.1 \leq j \leq N\right\}$. Further, Inn $W$ is isomorphic to a finite-index subgroup of $W$ in the case that $W$ is a graph product of primary cyclic groups.

We now justify two statements made in the introduction. First we demonstrate many interesting cases in which the equality Aut* $W=$ Aut $W$ holds. Recall from the introduction that, for each $1 \leq i \leq N$, we write $L_{i}$ for the link of $v_{i}$; that is, $L_{i}$ is the subgraph of $\Gamma$ generated by the vertices $\left\{v_{j} \in V \mid d\left(v_{i}, v_{j}\right)=1\right\}$. We shall also write $S_{i}$ for the star of $v_{i}$; that is, $S_{i}$ is the subgraph of $\Gamma$ generated by the vertices $\left\{v_{j} \in V \mid d\left(v_{i}, v_{j}\right) \leq 1\right\}$. The main result of [22] is that the automorphism group of a right-angled Artin group $W(\Gamma)$ is generated by the partial conjugations (see Section 3), automorphisms of the group induced by symmetries of the graph $\Gamma$, and "transvections". A right transvection is an automorphism $\tau_{i j}$ such that $i \neq j$, $L_{j} \subseteq S_{i}$ (in particular, $\left.d\left(v_{i}, v_{j}\right) \leq 2\right), \tau_{i j}\left(v_{j}\right)=v_{j} v_{i}$ and $\tau_{i j}\left(v_{k}\right)=v_{k}$ if $k \neq j$; left transvections are defined similarly. Of these generating automorphisms, only the transvections where $d\left(v_{i}, v_{j}\right)=2$ are not contained in Aut* $W$. Cases (2) and (3) of the lemma below follow immediately from this observation, , since they simply describe conditions on $\Gamma$ which ensure that $d\left(v_{i}, v_{j}\right)=1$ if $L_{j} \subseteq S_{i}$.

Lemma 2.8. If $W$ is a graph product of directly indecomposable cyclic groups, then Aut ${ }^{*} W=$ Aut $W$ in each of the following cases:

(1) $W$ is a graph product of primary cyclic groups;

(2) $W$ is a right-angled Artin group and $L_{i} \nsubseteq L_{j}$ for each pair of distinct nonadjacent vertices $v_{i}, v_{j} \in V$;

(3) $W$ is a right-angled Artin group and $\Gamma$ contains no vertices of valence less than two and no circuits of length less than 5.

Remark 2.9. Case (2) can be substantially generalized to groups that are not rightangled Artin groups.

Remark 2.10. In this remark we justify the statement that the subgroup Aut $^{1} W$ is, at least in some sense, well-understood. If $W_{\mathrm{ab}}$ denotes the abelianization of $W$, then Aut ${ }^{1} W$ is isomorphic to the image of Aut ${ }^{*} W$ under the natural map Aut $W \rightarrow$ Aut $W_{\mathrm{ab}}$. In particular, $\operatorname{Aut}^{1} W$ is finite in the case that $W$ is a graph product of primary cyclic groups. 


\section{A decomposition of $\mathrm{Aut}^{*} W$}

In this section we prove Theorem 1.2. Our proof is presented in three parts. In Subsection 3.1 we prove the following.

Theorem 3.1 (cf. [30]). If $W$ is a graph product of directly indecomposable cyclic groups, then Aut ${ }^{*} W=\operatorname{Aut}^{0} W \rtimes \operatorname{Aut}^{1} W$.

As noted in the introduction, Tits [30] proved Theorem 3.1 in the special case that $W$ is a right-angled Coxeter group. Although one could generalize the argument of [30] to prove Theorem 3.1 (see Remark 3.8), we take a different, but related, approach.

In Subsection 3.2 we prove the Restricted Alphabet Rewriting Lemma. This is the new technical tool which allows us to achieve many of our aims.

In Subsection 3.3 we complete our task, with the help of the Restricted Alphabet Rewriting Lemma, by proving the following.

Theorem 3.2. If $W$ is a graph product of directly indecomposable cyclic groups, then

$$
\text { Aut }^{0} W=\operatorname{Inn} W \rtimes \operatorname{Out}^{0} W .
$$

Before we begin, we describe an important set of generators for $\mathrm{Aut}^{0} W$. For $1 \leq i \leq N$ and $K$ a non-trivial connected component of $\Gamma \backslash S_{i}$, we write $\chi_{i K}$ for the automorphism of $W$ determined by

$$
\chi_{i K}\left(v_{j}\right)= \begin{cases}v_{i} v_{j} v_{i}^{-1} & \text { if } v_{j} \in K, \\ v_{j} & \text { if } v_{j} \notin K .\end{cases}
$$

Such an automorphism is called a partial conjugation with operating letter $v_{i}$ and domain $K$. We write $\mathcal{P}$ for the set of partial conjugations. Laurence [21], Theorem 4.1, proved that Aut $^{0} W$ is generated by $\mathcal{P}$.

3.1. A splitting of Aut $^{*} W$. In this section we prove that $\mathrm{Aut}^{*} W=\mathrm{Aut}^{0} W \rtimes$ Aut ${ }^{1} W$ (Theorem 3.1). We shall prove the result by exhibiting a retraction homomorphism Aut ${ }^{*} W \rightarrow$ Aut $^{1} W$ with kernel Aut ${ }^{0} W$.

Firstly we note that a conjugacy class in $W$ contains at most one element which can be written as a commuting product of generators (that is, only using generators from some complete subgraph). This can be seen as a consequence of Lemma 2.5 as follows: if $u, w \in W$ are commuting products of generators and they are conjugate in $W$, then $\operatorname{pr}_{\{v\}}(u)$ and $p r_{\{v\}}(w)$ are conjugate in $\langle v\rangle$, and hence equal, for each $v \in V$; thus $u$ and $w$ are commuting products of generators which use exactly the same generators and the same exponents of generators (modulo the orders of the respective generators). If $u \in W$ is conjugate to a commuting product of generators, then we write $[u]$ for the unique element of $W$ which can be written as a commuting product of generators. 
Let $\gamma \in$ Aut $^{*} W$. The definition of Aut ${ }^{*} W$ implies immediately that $\gamma(v)$ is conjugate to a commuting product of generators for each vertex $v \in V_{\Gamma}$. We define $r(\gamma): V \rightarrow W$ by the rule $v \mapsto[\gamma(v)]$.

Lemma 3.3. For each automorphism $\gamma \in$ Aut $^{*} W$, the map $r(\gamma): V \rightarrow W$ extends to an endomorphism of $W$.

Proof. It suffices to show that the relations used to define $W$ are 'preserved' by $r(\gamma)$.

Let $1 \leq i \leq N$ be such that $\boldsymbol{m}(i)<\infty$. Since the order of an element is preserved under automorphisms and conjugation, $\left(r(\gamma)\left(v_{i}\right)\right)^{\boldsymbol{m}\left(v_{i}\right)}=1$ and $r(\gamma)$ preserves the relation $v_{i}^{\boldsymbol{m}\left(v_{i}\right)}=1$.

Let $1 \leq j<k \leq N$ be such that $d\left(v_{j}, v_{k}\right)=1$. There exist $w \in W$ and $\Delta \in \operatorname{MCS}(\Gamma)$ and $a, b \in W(\Delta)$ such that $\gamma\left(v_{j}\right)=w a w^{-1}$ and $\gamma\left(v_{k}\right)=w b w^{-1}$. Recall that $W(\Delta)$ is an abelian group. Then

$$
\left(r(\gamma)\left(v_{j}\right)\right)\left(r(\gamma)\left(v_{k}\right)\right)\left(r(\gamma)\left(v_{j}\right)\right)^{-1}\left(r(\gamma)\left(v_{k}\right)\right)^{-1}=a b a^{-1} b^{-1}=1 .
$$

Thus $r(\gamma)$ preserves the relation $v_{j} v_{k} v_{j}^{-1} v_{k}^{-1}=1$.

We abuse notation by writing $r(\gamma): W \rightarrow W$ for the endomorphism determined by $r(\gamma): V \rightarrow W$.

Lemma 3.4. For each automorphism $\delta \in$ Aut $^{*} W$ and $\Delta \in \operatorname{MCS}(\Gamma)$ and $a \in W(\Delta)$, we have $r(\delta)(a)=[\delta(a)]$.

Proof. We have $a=d_{1}^{\epsilon_{1}} \ldots d_{q}^{\epsilon_{q}}$ for some vertices $d_{1}, \ldots, d_{q}$ in $\Delta$ and some integers $\epsilon_{1}, \ldots, \epsilon_{q}$. By the definition of Aut $^{*} W$, there exist $w \in W$ and $\Theta \in \operatorname{MCS}(\Gamma)$ such that $\delta(W(\Delta))=w W(\Theta) w^{-1}$. Hence there exist $t_{1}, \ldots, t_{q} \in W(\Theta)$ such that $\delta\left(d_{i}\right)=w t_{i} w^{-1}$ for each $1 \leq i \leq q$. Then

$$
\begin{aligned}
r(\delta)(a) & =r(\delta)\left(d_{1}\right)^{\epsilon_{1}} \ldots r(\delta)\left(d_{q}\right)^{\epsilon_{q}} \\
& =t_{1}^{\epsilon_{1}} \ldots t_{q}^{\epsilon_{q}}=\left[w t_{1}^{\epsilon_{1}} w^{-1} \ldots w t_{q}^{\epsilon_{q}} w^{-1}\right]=[\delta(a)] .
\end{aligned}
$$

as required.

Lemma 3.5. For each pair of automorphisms $\gamma, \delta \in$ Aut $^{*} W$, we have $r(\delta \gamma)=$ $r(\delta) r(\gamma)$.

Proof. Let $\gamma, \delta \in$ Aut $^{*} W$ and let $1 \leq i \leq N$. There exist $\Delta \in \operatorname{MCS}(\Gamma)$ and $a \in W(\Delta)$ and $w_{1} \in W$ such that $\gamma\left(v_{i}\right)=w_{1} a w_{1}^{-1}$. There exist $\Theta \in \operatorname{MCS}(\Gamma)$, $b \in W(\Theta)$ and $w_{2} \in W$ such that $\delta(W(\Delta))=w_{2} W(\Theta) w_{2}^{-1}$ and $\delta(a)=w_{2} b w_{2}^{-1}$. By Lemma 3.4 we have that $r(\delta)(a)=b$. Then

$$
r(\delta \gamma)\left(v_{i}\right)=\left[\delta \gamma\left(v_{i}\right)\right]=\left[\delta\left(w_{1}\right) w_{2} b w_{2}^{-1} \delta\left(w_{1}\right)^{-1}\right]=b=r(\delta)(a)=r(\delta) r(\gamma)\left(v_{i}\right),
$$

as required. 
Lemma 3.6. For each $\gamma \in$ Aut $^{*} W, r(\gamma) \in \operatorname{Aut}^{1} W$.

Proof. Let $\gamma \in$ Aut $^{*} W$. By Lemma 3.5, $r\left(\gamma^{-1}\right) r(\gamma)=r\left(\gamma^{-1} \circ \gamma\right)=r(i d)=i d$ and $r(\gamma)$ is an automorphism of $W$. It is clear from the definitions that $r(\gamma) \in \operatorname{Aut}^{1} W$.

Proposition 3.7. The map $r$ is a retraction homomorphism Aut ${ }^{*} W \rightarrow$ Aut $^{1} W$ with kernel Aut $^{0} W$.

Proof. By Lemmas 3.5 and 3.6, $r$ is a homomorphism Aut ${ }^{*} W \rightarrow$ Aut $^{1} W$. It is clear from the definitions that $r$ restricts to the identity map on Aut ${ }^{1} W$ and $r$ has kernel $\mathrm{Aut}^{0} W$.

Remark 3.8 (Tits' approach and Theorem 3.1). For each $\Delta \in \operatorname{MCS}(\Gamma)$, we may consider $W(\Delta)$ as a subgroup of $W_{\mathrm{ab}}$. Then the union

$$
G=\bigcup_{\Delta \in \operatorname{MCS}(\Gamma)} W(\Delta) \subset W_{\mathrm{ab}}
$$

is a groupoid in the usual way. In case $W$ is a right-angled Coxeter group, Tits [30] identifies Aut ${ }^{1} W$ with the groupoid automorphisms Aut $G$ of $G$ and constructs a section of the obvious homomorphism Aut ${ }^{*} W \rightarrow$ Aut $G$. This identification carries over in the case that $W$ is an arbitrary graph product of directly indecomposable groups and a section of the homomorphism Aut ${ }^{*} W \rightarrow$ Aut $G$ is defined similarly.

3.2. The Restricted Alphabet Rewriting Lemma. For a subgraph $\Omega \subseteq \Gamma$, we write $\operatorname{pr}_{\Omega}$ for the retraction homomorphism $W \rightarrow W(\Omega)$ and $\mathcal{P}_{\Omega}:=\left\{\chi_{i} \in \mathcal{P} \mid v_{i} \in \Omega\right\}$. For $\phi \in$ Aut $^{0} W$ and $w_{1}, \ldots, w_{N} \in W$ such that $\phi\left(v_{i}\right)=w_{i} v_{i} w_{i}^{-1}$ for each $1 \leq i \leq N$, we write $\phi_{\Omega}$ for the map $V \rightarrow W$ defined by

$$
v_{i} \mapsto \operatorname{pr}_{\Omega}\left(w_{i}\right) \cdot v_{i} \cdot \operatorname{pr}_{\Omega}\left(w_{i}\right)^{-1} \quad \text { for each } 1 \leq i \leq N .
$$

We shall show that $\phi_{\Omega}$ extends to an automorphism of $W$, also denoted by $\phi_{\Omega}$. In fact, the following theorem and its immediate corollary hold.

Theorem 3.9. For each subgraph $\Omega \subseteq \Gamma$, the map $\phi \mapsto \phi_{\Omega}$ is a retraction homomorphism Aut $^{0} W \rightarrow\left\langle\mathcal{P}_{\Omega}\right\rangle$.

Corollary 3.10 (The Restricted Alphabet Rewriting Lemma). If $\phi \in \mathrm{Aut}^{0} W$ and there exist $z_{1}, \ldots, z_{N} \in W(\Omega)$ such that $\phi\left(v_{j}\right)=z_{j} v_{j} z_{j}^{-1}$ for each $1 \leq j \leq N$, then any word for $\phi$ in the alphabet $\mathcal{P}^{ \pm 1}$ may be rewritten as a word in the alphabet $\mathcal{P}_{\Omega}^{ \pm 1}$ ( a word which still spells $\phi$ but only uses partial conjugations whose operating letter is in $\Omega$, or their inverses) by simply omitting those generators not in $\mathcal{P}_{\Omega}^{ \pm 1}$. 
Remark 3.11. The Restricted Alphabet Rewriting Lemma implies that the subgroup $\left\langle\mathcal{P}_{\Omega}\right\rangle$ is convex in Aut $^{0} W$. That is, for each automorphism $\phi \in\left\langle\mathcal{P}_{\Omega}\right\rangle$, the shortest ways to spell $\phi$ in the alphabet $\mathcal{P}^{ \pm 1}$ only use letters from the alphabet $\mathcal{P}_{\Omega}^{ \pm 1}$.

This subsection is devoted to proving Theorem 3.9. We also illustrate the power of The Restricted Alphabet Rewriting Lemma by proving that $\mathcal{P}$ is a minimal generating set for Aut $^{0} W$. Throughout, we fix a subgraph $\Omega \subseteq \Gamma$.

Lemma 3.12. The map $\phi \mapsto \phi_{\Omega}$ is well defined.

Proof. Let $\phi \in$ Aut $^{0} W$ and $w_{1}, \ldots, w_{N} \in W$ and $u_{1}, \ldots, u_{N} \in W$ be such that $\phi\left(v_{i}\right)=w_{i} v_{i} w_{i}^{-1}=u_{i} v_{i} u_{i}^{-1}$ for each $1 \leq i \leq N$. Fix $1 \leq i \leq N$. We must show that $\operatorname{pr}_{\Omega}\left(w_{i}\right) \cdot v_{i} \cdot \operatorname{pr}_{\Omega}\left(w_{i}\right)^{-1}=\operatorname{pr}_{\Omega}\left(u_{i}\right) \cdot v_{i} \cdot \operatorname{pr}_{\Omega}\left(u_{i}\right)^{-1}$. Since $w_{i} v_{i} w_{i}^{-1}=u_{i} v_{i} u_{i}^{-1}$, we have that $u_{i}^{-1} w_{i}$ is in the centralizer of $v_{i}$. Recall that the centralizer of $v_{i}$ is generated by $S_{i}$ (Lemma 2.4). Thus there exists $z_{i} \in\left\langle S_{i}\right\rangle$ such that $w_{i}=u_{i} z_{i}$. Since $\operatorname{pr}_{\Omega}\left(S_{i}\right) \subseteq S_{i} \cup\{\mathrm{id}\}, \operatorname{pr}_{\Omega}\left(z_{i}\right) \in\left\langle S_{i}\right\rangle$ and we have

$$
\begin{aligned}
\operatorname{pr}_{\Omega}\left(w_{i}\right) \cdot v_{i} \cdot \operatorname{pr}_{\Omega}\left(w_{i}\right)^{-1} & =\operatorname{pr}_{\Omega}\left(u_{i} z_{i}\right) \cdot v_{i} \cdot \operatorname{pr}_{\Omega}\left(u_{i} z_{i}\right)^{-1} \\
& =\operatorname{pr}_{\Omega}\left(u_{i}\right) \cdot \operatorname{pr}_{\Omega}\left(z_{i}\right) \cdot v_{i} \cdot \operatorname{pr}_{\Omega}\left(z_{i}\right)^{-1} \cdot \operatorname{pr}_{\Omega}\left(u_{i}\right)^{-1} \\
& =\operatorname{pr}_{\Omega}\left(u_{i}\right) \cdot v_{i} \cdot \operatorname{pr}_{\Omega}\left(u_{i}\right)^{-1}
\end{aligned}
$$

Lemma 3.13. For each $\phi \in \operatorname{Aut}^{0} W$, the map $\phi_{\Omega}: V \rightarrow W$ extends to a homomorphism $\phi_{\Omega}: W \rightarrow W$.

Proof. Let $\phi \in \operatorname{Aut}^{0} W$ and $w_{1}, \ldots, w_{N} \in W$ be such that $\phi\left(v_{i}\right)=w_{i} v_{i} w_{i}^{-1}$ for each $1 \leq i \leq N$. We must show that the map $\phi_{\Omega}$ 'preserves' the defining relations of $W$.

Let $1 \leq i \leq N$. Since $\phi_{\Omega}\left(v_{i}\right)$ is conjugate to $v_{i}$, it has the same order as $v_{i}$ and the relation $v_{i}^{\boldsymbol{m}(i)}$ is preserved.

Let $1 \leq i<j \leq N$ be such that $v_{i}$ and $v_{j}$ are adjacent. Since $\phi \in \operatorname{Aut}^{0} W$, it follows that there exists $w \in W$ such that $\phi\left(v_{i}\right)=w v_{i} w^{-1}$ and $\phi\left(v_{j}\right)=w v_{j} w^{-1}$. Then $w_{i}=w z_{i}$ for some $z_{i} \in\left\langle S_{i}\right\rangle$ and $w_{j}=w z_{j}$ for some $z_{j} \in\left\langle S_{j}\right\rangle$. So $w_{i}^{-1} w_{j}=z_{i}^{-1} z_{j}$ and $\operatorname{pr}_{\Omega}\left(w_{i}^{-1}\right) \cdot \operatorname{pr}_{\Omega}\left(w_{j}\right)=\operatorname{pr}_{\Omega}\left(z_{i}^{-1}\right) \cdot \operatorname{pr}_{\Omega}\left(z_{j}\right)$. We have

$$
\begin{aligned}
& \phi_{\Omega}\left(v_{i}\right) \cdot \phi_{\Omega}\left(v_{j}\right) \cdot \phi_{\Omega}\left(v_{i}\right)^{-1} \cdot \phi_{\Omega}\left(v_{i}\right)^{-1}=\operatorname{pr}_{\Omega}\left(w_{i}\right) \cdot v_{i} \cdot \operatorname{pr}_{\Omega}\left(w_{i}^{-1}\right) \cdot \operatorname{pr}_{\Omega}\left(w_{j}\right) \cdot v_{j} \cdot \operatorname{pr}_{\Omega}\left(w_{j}\right)^{-1} \\
& \quad \operatorname{pr}_{\Omega}\left(w_{i}\right) \cdot v_{i}^{-1} \cdot \operatorname{pr}_{\Omega}\left(w_{i}\right)^{-1} \cdot \operatorname{pr}_{\Omega}\left(w_{j}\right) \cdot v_{j}^{-1} \cdot \operatorname{pr}_{\Omega}\left(w_{j}\right)^{-1} \\
&= \operatorname{pr}_{\Omega}\left(w_{i}\right) \cdot v_{i} \cdot \operatorname{pr}_{\Omega}\left(z_{i}^{-1}\right) \cdot \operatorname{pr}_{\Omega}\left(z_{j}\right) \cdot v_{j} \cdot \operatorname{pr}_{\Omega}\left(z_{j}\right)^{-1} \\
& \quad \cdot \operatorname{pr}_{\Omega}\left(z_{i}\right) \cdot v_{i}^{-1} \cdot \operatorname{pr}_{\Omega}\left(z_{i}\right)^{-1} \cdot \operatorname{pr}_{\Omega}\left(z_{j}\right) \cdot v_{j}^{-1} \cdot \operatorname{pr}_{\Omega}\left(w_{j}\right)^{-1} \\
&=\operatorname{pr}_{\Omega}\left(w_{i}\right) \cdot \operatorname{pr}_{\Omega}\left(z_{i}^{-1}\right) \cdot v_{i} v_{j} v_{i}^{-1} v_{j}^{-1} \cdot \operatorname{pr}_{\Omega}\left(z_{j}\right) \cdot \operatorname{pr}_{\Omega}\left(w_{j}\right)^{-1}
\end{aligned}
$$




$$
\begin{aligned}
& =\operatorname{pr}_{\Omega}\left(w_{i} z_{i}^{-1}\right) \cdot 1 \cdot \operatorname{pr}_{\Omega}\left(w_{j} z_{j}^{-1}\right)^{-1} \\
& =\operatorname{pr}_{\Omega}(w) \cdot \operatorname{pr}_{\Omega}(w)^{-1} \\
& =1
\end{aligned}
$$

and the relation $v_{i} v_{j} v_{i}^{-1} v_{j}^{-1}$ is preserved.

Lemma 3.14. For $\phi, \theta \in \operatorname{Aut}^{0} W,(\phi \circ \theta)_{\Omega}=\phi_{\Omega} \circ \theta_{\Omega}$.

Proof. Fix $1 \leq i \leq N$. It suffices to show that $(\theta \circ \phi)_{\Omega}\left(v_{i}\right)=\left(\theta_{\Omega} \circ \phi_{\Omega}\right)\left(v_{i}\right)$. Write $V^{*}$ for the set of (not necessarily reduced) words in the alphabet $V^{ \pm 1}$. Let $\mathfrak{w}_{1}, \ldots, \mathfrak{w}_{N} \in V^{*}$ and $U_{1}, \ldots, u_{N} \in V^{*}$ be such that $\phi\left(v_{j}\right)=\mathfrak{w}_{j} v_{j} \mathfrak{W}_{j}^{-1}$ (with equality in $W$ ) and $\theta\left(v_{j}\right)=u_{j} v_{j} u_{j}^{-1}$ (with equality in $W$ ) for each $1 \leq j \leq N$. Let $\mathcal{T}_{i}$ be the word constructed from $W_{i}$ as follows:

(1) for each $1 \leq j \leq N$ and $\delta= \pm 1$, replace each occurrence of $v_{j}^{\delta}$ by $U_{j} v_{j}^{\delta} U_{j}^{-1}$;

(2) append the word $\mathcal{U}_{i}$ to the resulting word;

(3) omit those letters not in $\Omega$ from the resulting word.

It is clear that $\mathcal{T}_{i}=\operatorname{pr}_{\Omega}\left(\theta\left(\mathcal{W}_{i}\right) U_{i}\right)$ and hence $(\theta \phi)_{\Omega}\left(v_{i}\right)=\mathcal{T}_{i} v_{i} \mathcal{T}_{i}^{-1}$ (with equality in $W$ ). Observe the following in the construction of $\mathcal{T}_{i}$ :

(OB1) if $v_{j} \notin \Omega$, then each occurrence of $v_{j}^{\delta}$ in $W_{j}$ is eventually replaced by the word $\operatorname{pr}_{\Omega}\left(U_{j}\right) \cdot \operatorname{pr}_{\Omega}\left(U_{j}\right)^{-1}$, which is, of course, trivial in $W$;

(OB2) if $v_{j} \in \Omega$, then each occurrence of $v_{j}^{\delta}$ in $W_{j}$ is eventually replaced by the word $\operatorname{pr}_{\Omega}\left(U_{j}\right) \cdot v_{j}^{\delta} \cdot \operatorname{pr}_{\Omega}\left(U_{j}\right)^{-1}$.

Let $\mathcal{T}_{i}^{\prime}$ be the word constructed from $W_{i}$ as follows:

(1) for each $1 \leq j \leq N$ such that $v_{j} \notin \Omega$ and each $\delta= \pm 1$, omit each occurrence of $v_{j}^{\delta}$;

(2) for each $1 \leq j \leq N$ such that $v_{j} \in \Omega$ and each $\delta= \pm 1$, replace each occurrence of $v_{j}^{\delta}$ by $\operatorname{pr}_{\Omega}\left(\bar{U}_{j}\right) \cdot v_{j}^{\delta} \cdot \operatorname{pr}_{\Omega}\left(U_{j}\right)^{-1}$

(3) append the word $\operatorname{pr}_{\Omega}\left(U_{i}\right)$ to the resulting word.

It follows from (OB1) and (OB2) that $\mathcal{T}_{i}^{\prime}=\mathcal{T}_{i}$ (with equality in $W$ ). It is clear from the construction of $\mathcal{T}_{i}^{\prime}$ that $\mathcal{T}_{i}^{\prime}=\left(\theta_{\Omega} \circ \operatorname{pr}_{\Omega}\left(W_{i}\right)\right) \cdot \operatorname{pr}_{\Omega}\left(U_{i}\right)$ (with equality in $W$ ). We calculate the following (with all equalities in $W$ ):

$$
\begin{aligned}
(\theta \phi)_{\Omega}\left(v_{i}\right) & =\mathcal{T}_{i} v_{i} \mathcal{T}_{i}^{-1} \\
& =\widetilde{T}_{i}^{\prime} v_{i}\left(\mathcal{T}_{i}^{\prime}\right)^{-1} \\
& =\left(\theta_{\Omega} \circ \operatorname{pr}_{\Omega}\left(W_{i}\right)\right) \cdot \operatorname{pr}_{\Omega}\left(U_{i}\right) \cdot v_{i} \cdot \operatorname{pr}_{\Omega}\left(\mathcal{U}_{i}\right)^{-1} \cdot\left(\theta_{\Omega} \circ \operatorname{pr}_{\Omega}\left(W_{i}\right)\right)^{-1} \\
& =\theta_{\Omega}\left(\operatorname{pr}_{\Omega}\left(W_{i}\right)\right) \cdot \theta_{\Omega}\left(v_{i}\right) \cdot \theta_{\Omega}\left(\operatorname{pr}_{\Omega}\left(\mathcal{W}_{i}\right)^{-1}\right) \\
& =\theta_{\Omega}\left(\operatorname{pr}_{\Omega}\left(W_{i}\right) \cdot v_{i} \cdot \operatorname{pr}_{\Omega}\left(W_{i}\right)^{-1}\right) \\
& =\theta_{\Omega} \circ \phi_{\Omega}\left(v_{i}\right) .
\end{aligned}
$$


We now prove the main result of the subsection.

Proof of Theorem 3.9. By Lemma 3.14, $\left(\phi^{-1}\right)_{\Omega} \circ \phi_{\Omega}=\left(\phi^{-1} \circ \phi\right)_{\Omega}=i d_{\Omega}=i d$ and $\phi_{\Omega}$ is an automorphism of $W$. So the map $\phi \mapsto \phi_{\Omega}$ is a map $\operatorname{Aut}^{0} W \rightarrow \operatorname{Aut}^{0} W$. It also follows from Lemma 3.14 that $\phi \mapsto \phi_{\Omega}$ is a homomorphism $\operatorname{Aut}^{0} W \rightarrow$ Aut $^{0} W$. It is clear from the definitions that

$$
\left(\chi_{i K}\right)_{\Omega}= \begin{cases}\chi_{i K} & \text { if } \chi_{i K} \in \mathcal{P}_{\Omega}, \\ \text { id } & \text { if } \chi_{i K} \notin \mathcal{P}_{\Omega}\end{cases}
$$

It follows that $\phi \mapsto \phi_{\Omega}$ is a retraction homomorphism Aut $^{0} W \rightarrow\left\langle\mathcal{P}_{\Omega}\right\rangle$.

We conclude this subsection by noting the following immediate application of The Restricted Alphabet Rewriting Lemma.

Corollary 3.15. The set $\mathcal{P}$ is a minimal generating set for $\mathrm{Aut}^{0} W$.

Proof. Let $\chi_{i K} \in \mathcal{P}$ and let $\mathcal{U}$ be a word in the alphabet $\mathcal{P}^{ \pm 1}$ such that $\mathcal{U}=\chi_{i K}$ (with equality in $\mathrm{Aut}^{0} W$ ). It follows from the Restricted Alphabet Rewriting Lemma that, simply by omitting some letters, $\mathcal{U}$ may be rewritten as a word $\mathcal{U}^{\prime}$ in the alphabet

$$
\left\{\chi_{i} Q \mid Q \text { a connected component of } \Gamma \backslash S_{i}\right\}^{ \pm 1} \text {. }
$$

But the letters in $U^{\prime}$ commute pairwise, so we must have that $\chi_{i K}$ appears with exponent sum 1 in $\mathcal{U}^{\prime}$ and hence also with exponent sum 1 in $\mathcal{U}$. Thus no word in the alphabet

$$
\left(\mathcal{P} \backslash\left\{\chi_{i K}\right\}\right)^{ \pm 1}
$$

can spell $\chi_{i K}$

3.3. A splitting of $\mathbf{A u t}^{0} \boldsymbol{W}$. In this subsection we define a subset $\mathscr{P}^{0} \subset \mathcal{P}$ which generates a complement of Inn $W$ in $\operatorname{Aut}^{0} W$. Thus we show that $\left\langle\mathcal{P}^{0}\right\rangle \cong$ Out $^{0} W$ and Aut $^{0} W=$ Inn $W \rtimes$ Out $^{0} W$ (Theorem 3.2). Informally, one might understand the construction of $\mathcal{P}^{0}$ from $\mathcal{P}$ as removing 'just enough' automorphisms to prevent the elements of $\left(\mathscr{P}^{0}\right)^{ \pm 1}$ from spelling a non-trivial inner-automorphism.

Let $\mathcal{I}$ denote the following set of inner automorphisms

$$
\mathcal{I}=\left\{w \mapsto v_{i} w v_{i}^{-1} \mid 1 \leq i \leq N \text { and } \Gamma \backslash S_{i} \neq \emptyset\right\}
$$

It is clear that $\mathcal{I}$ generates Inn $W$. The commuting product $\prod_{K} \chi_{i K}$, taken over all nontrivial connected components $K$ of $\Gamma \backslash S_{i}$, is the inner automorphism $(w \mapsto$ $\left.v_{i} w v_{i}^{-1}\right) \in \mathcal{I}$. If, starting with $\mathcal{P}$, we remove one $\chi_{i K}$ for each $i$ such that $\Gamma \backslash S_{i} \neq \emptyset$, then the union of the resulting set and $\mathcal{I}$ is a generating set for $\operatorname{Aut}^{0} W$. We now do so systematically. 
Definition 3.16 $\left(\mathscr{P}^{0}\right)$. For each $1 \leq i \leq N$ such that $\Gamma \backslash S_{i} \neq \emptyset$, let $j_{i}$ be minimal such that $v_{j_{i}} \in \Gamma \backslash S_{i}$. Define

$$
\mathcal{P}^{0}:=\left\{\chi_{i K} \in \mathcal{P} \mid v_{j_{i}} \notin K\right\} .
$$

Remark 3.17. Observe the following properties of $\mathcal{P}^{0}$ :

(1) As in Corollary 3.15, the Restricted Alphabet Rewriting Lemma may be used to show that the set $\mathcal{I} \cup \mathcal{P}^{0}$ is a minimal generating set for $\mathrm{Aut}^{0} W$.

(2) For each $1 \leq i \leq N$, either $\Gamma \backslash S_{i}=\emptyset$ or the set $\mathcal{P} \backslash \mathcal{P}^{0}$ contains exactly one element of the form $\chi_{i K}$.

(3) If $\chi_{i K} \in \mathcal{P}^{0}$, then $v_{1} \notin K$. If $\chi_{i K} \in \mathcal{P}^{0}$ and $d\left(v_{1}, v_{i}\right) \leq 1$, then $v_{2} \notin K$. In general, if $\chi_{i K} \in \mathscr{P}^{0}$ and $d\left(v_{j}, v_{i}\right) \leq 1$ for each $1 \leq j \leq k$, then $v_{k+1} \notin K$.

(4) The set $\mathcal{P}^{0}$ depends on the ordering of $V$ defined by the indexing, but the isomorphism type of $\mathrm{Out}^{0} \mathrm{~W}$ does not. For the following proof, the ordering is unimportant.

Lemma 3.18. $\left\langle\mathscr{P}^{0}\right\rangle \cap \operatorname{Inn} W=\{\operatorname{id}\}$.

Proof. For each $1 \leq i \leq N$, write $S_{i}:=\left\{\chi_{j K} \in \mathcal{P}^{0} \mid v_{j} \in S_{i}\right\}$ and write $\mathcal{S}:=\cap_{i=1}^{N} \delta_{i}$. Suppose that $\phi \in \operatorname{Inn} W \cap$ Out $^{0} W$, say $\phi\left(v_{j}\right)=w v_{j} w^{-1}$ for each $1 \leq j \leq N$. We shall use induction to show that $\phi \in\langle\mathcal{S}\rangle$. We shall then show that $S=\emptyset$.

Since $\phi \in\left\langle\mathcal{P}^{0}\right\rangle, \phi$ may be written as a word $\Phi_{0}$ in the alphabet $\left(\mathcal{P}^{0}\right)^{ \pm 1}$. By Remark 3.17 (3), each element of $\mathscr{P}^{0}$ acts trivially on $v_{1}$. It follows from The Deletion Condition that $w$ is in the centralizer of $v_{1}$. By Lemma 2.4, $w \in W\left(S_{1}\right)$. By the Restricted Alphabet Rewriting Lemma, $\phi$ may be written as a product $\Phi_{1}$ in the alphabet $\delta_{1}^{ \pm 1}$ (starting with $\Phi_{0}$, delete those letters not in $S_{1}^{ \pm 1}$ ). Now let $i$ be an integer such that $1 \leq i<N$ and suppose that $\phi$ may be written as a product $\Phi_{i}$ in the alphabet $\left(\delta_{1} \cap \cdots \cap \delta_{i}\right)^{ \pm 1}$. By Remark 3.17 (3), each element of $\delta_{1} \cap \cdots \cap S_{i}$ acts trivially on $v_{i+1}$. It follows that $w$ is in the centralizer of $v_{i+1}$. Hence $w \in W\left(S_{i+1}\right)$. By the Restricted Alphabet Rewriting Lemma, $\phi$ may be written as a product $\Phi_{i+1}$ in the alphabet $\left(S_{1} \cap \cdots \cap S_{i} \cap S_{i+1}\right)^{ \pm 1}$ (starting with $\Phi_{i}$, delete those letters not in $S_{i+1}^{ \pm 1}$ ). By induction we have that $\phi$ may be written as a product $\Phi_{N}$ in the alphabet $\delta^{ \pm 1}$.

Now $\chi_{j K} \in S$ if and only if $v_{j}$ is adjacent to each vertex in $\Gamma$. But for such $v_{j}$, $\Gamma \backslash S_{j}=\emptyset$ and $\mathcal{P}$ (and hence $\mathcal{P}^{0}$ ) contains no partial conjugations with operating letter $v_{j}$. Thus $\delta=\emptyset, \Phi_{N}$ is the empty word and $\phi$ is the trivial automorphism.

Proof of Theorem 3.2. This follows immediately from Lemma 3.18, Remark 3.17 (1) and the fact that Inn $W$ is a normal subgroup of $\operatorname{Aut}^{0} W$.

3.4. An application to group extensions. The authors of [14] considered the special case that $W$ is a right-angled Coxeter group. They give sufficient conditions (distinct 
from those below) for Aut $^{0} W$ to split as Inn $W \rtimes \operatorname{Aut}^{0} W / \operatorname{Inn} W$, and show that this splitting is compatible with the splitting Aut $W=$ Aut $^{0} W \rtimes \operatorname{Aut}^{1} W$ of [30]. It follows that their conditions ensure Aut $W=\operatorname{Inn} W \rtimes$ Out $W$ and that each extension of $W$ is a split extension. We now follow an analogous program for graph products of directly indecomposable cyclic groups. We give conditions, distinct from those in [14], which ensure that each group extension of $W$ is a split extension.

For each $1 \leq i \leq N$, we write $\Delta_{i}:=\left\{v_{j} \in V \mid S_{i}=S_{j}\right\}$ (note that $\Delta_{i}$ is a complete subgraph for each $1 \leq i \leq N$ ).

Lemma 3.19. If $W$ is a graph product of directly indecomposable cyclic groups and $\phi\left(W\left(\Delta_{i}\right)\right)=W\left(\Delta_{i}\right)$ for each $1 \leq i \leq N$ and $\phi \in$ Aut $^{1} W$, then the splittings of Theorems 3.1 and 3.2 are compatible; that is, one may write

$$
\text { Aut }^{*} W=\operatorname{Inn} W \rtimes\left(\mathrm{Out}^{0} W \rtimes \mathrm{Aut}^{1} W\right) \cong \operatorname{Inn} W \rtimes \mathrm{Out}^{*} W .
$$

Proof. Let $\phi \in$ Aut $^{1} W$ and $\theta \in$ Out $^{0} W$. If $\theta$ is not the identity, then the product $\phi \theta$ is not an inner automorphism since it acts non-trivially on the set of conjugacy classes of cyclically reduced involutions in $W$. If $\theta$ is the identity but $\phi$ is not, then the product $\phi \theta=\phi$ is not an inner automorphism by Lemma 3.18. Thus, to show that Inn $W \cap$ Out $^{*} W=\{\mathrm{id}\}$ and hence the result, it suffices to show that Out $^{*} W=$ Out $^{0} W$.Aut ${ }^{1} W$.

Let $\phi \in$ Aut $^{1} W$ and $\chi_{i K} \in \mathcal{P}^{0}$. It suffices to show that $\phi^{-1} \chi_{i K} \phi \in\left\langle\mathcal{P}^{0}\right\rangle$. Let $1 \leq j \leq N$. If $v_{j} \notin K$, then $\Delta_{j} \cap K=\emptyset$ and the support of $\phi\left(v_{j}\right)$ is disjoint from $K$. Hence

$$
\phi^{-1} \chi_{i K} \phi\left(v_{j}\right)=\phi^{-1}\left(\chi_{i K}\left(\phi\left(v_{j}\right)\right)\right)=\phi^{-1} \phi\left(v_{j}\right)=v_{j} .
$$

If $v_{j} \in K$, then $\Delta_{j} \subseteq K$ and the support of $\phi\left(v_{j}\right)$ is contained in $K$. Hence $\phi^{-1} \chi_{i K} \phi\left(v_{j}\right)=\phi^{-1}\left(\chi_{i K}\left(\phi\left(v_{j}\right)\right)\right)=\phi^{-1}\left(v_{i} \phi\left(v_{j}\right) v_{i}^{-1}\right)=\phi^{-1}\left(v_{i}\right) v_{j}\left(\phi^{-1}\left(v_{i}\right)\right)^{-1}$.

Thus we have

$$
\phi^{-1} \chi_{i K} \phi\left(v_{j}\right)= \begin{cases}v_{j} & \text { if } v_{j} \notin K, \\ \phi^{-1}\left(v_{i}\right) v_{j}\left(\phi^{-1}\left(v_{i}\right)\right)^{-1} & \text { if } v_{j} \in K .\end{cases}
$$

By hypothesis, $\phi^{-1}\left(v_{i}\right) \in W\left(\Delta_{i}\right)$. For each $v_{\ell} \in \Delta_{i}$, the least element of $\Gamma \backslash S_{i}$ is also the least element of $\Gamma \backslash S_{\ell}$ and, since $\chi_{i K} \in \mathcal{P}^{0}$, we have $\chi_{\ell K} \in \mathcal{P}^{0}$. Thus $\phi^{-1} \chi_{i K} \phi$ may be written as a product of elements in $\left(\mathscr{P}^{0}\right)^{ \pm 1}$.

Recall that the center of $W$ is the special subgroup generated by those vertices adjacent to every other vertex. Recall also that Lemma 2.8 gives sufficient conditions for the equality Aut* $W=$ Aut $W$.

Corollary 3.20. If $W$ is a graph product of directly indecomposable cyclic groups and the conditions 
(1) W has trivial center,

(2) Aut $^{*} W=$ Aut $W$,

(3) $\phi\left(W\left(\Delta_{i}\right)\right)=W\left(\Delta_{i}\right)$ for each $1 \leq i \leq N$ and each $\phi \in \operatorname{Aut}^{1} W$

are satisfied, then each group extension of the form $1 \rightarrow W \rightarrow E \rightarrow G \rightarrow 1$ is a split extension.

Proof. Conditions (2) and (3) and Lemma 3.19 give that Aut $W=\operatorname{Inn} W \rtimes$ Out $W$. Thus each homomorphism $\psi: G \rightarrow$ Out $W$ lifts to a homomorphism $\hat{\psi}: G \rightarrow$ Aut $W$ and hence determines a semidirect product $W \rtimes_{\hat{\psi}} G$. Condition (1) of the hypothesis ensures that there is exactly one extension of $G$ by $W$ (up to equivalence) corresponding to any homomorphism $\psi: G \rightarrow$ Out $W$ [3], Corollary IV.6.8, p. 106.

\section{The structure and cardinality of $\mathrm{Out}^{0} \mathrm{~W}$}

We now turn our attention to $\operatorname{Out}^{0} W$. In subsection 4.1 we prove Theorem 1.4 by examining the combinatorics of partial conjugations. In Section 4.2 we describe a number of applications of the theorem.

4.1. The proof of Theorem 1.4. Theorem 1.4 is essentially a consequence of the ways in which the connected components of $\Gamma \backslash S_{i}$ and $\Gamma \backslash S_{j}$ may interact for $i \neq j$. In this subsection we record some graph-theoretic lemmas which help us understand these interactions, and eventually prove the result. First though, we shall describe a construction and record some properties of the construction which allow us to make a strong assumption about $\Gamma$ without loss of generality.

Construction 4.1. Write $\left(\Gamma^{+}, m^{+}\right)$for the labeled graph obtained from $(\Gamma, m)$ as follows:

(1) introduce a new vertex $v_{0}$ and extend $\boldsymbol{m}$ to a function $\boldsymbol{m}^{+}$with domain $\{0,1,2, \ldots, N\}$ by making a choice

$$
\boldsymbol{m}(0) \in\left\{p^{\alpha} \mid p \text { prime and } \alpha \in \mathbb{N}\right\} \cup\{\infty\} ;
$$

(2) add an edge from $v_{0}$ to $v_{i}$ for each $1 \leq i \leq N$.

Write $W^{+}:=W\left(\Gamma^{+}, \boldsymbol{m}^{+}\right)$and write $S_{i}^{+}$for the star of $v_{i}$ in $\Gamma^{+}$.

It follows from Lemma 2.7 that the construction is easily understood in a group theoretic way: passing from $W$ to $W^{+}$corresponds to passing from the group $W$ to the group $W^{+} \cong W \times C$, where $C$ is the cyclic group of order $\boldsymbol{m}(0)$.

Lemma 4.2. Let $W^{+}$and $\Gamma^{+}$be as in Construction 4.1. Then 
(1) Aut $^{0} W \cong \operatorname{Aut}^{0} W^{+}$;

(2) Out $^{0} W \cong \mathrm{Out}^{0} W^{+}$;

(3) $\Gamma^{+}$has a SIL if and only if $\Gamma$ has a SIL.

Proof. For each $1 \leq i \leq N$, the connected components of $\Gamma \backslash S_{i}$ are identical to the connected components of $\Gamma^{+} \backslash S_{i}^{+}$. The subgraph $\Gamma^{+} \backslash S_{0}^{+}$is empty. Since $\mathcal{P}$ generates Aut ${ }^{0} W$, it follows that Aut $^{0} W \cong$ Aut $^{0} W^{+}$. It also follows from the construction of $\mathcal{P}^{0}$ that $\mathrm{Out}^{0} W \cong \mathrm{Out}^{0} W^{+}$. Property (3) is immediate from the definitions.

Thus we do not limit the scope of our study by assuming that there exists a vertex $v \in V_{\Gamma}$ which is adjacent to every other vertex. We shall make this assumption for the remainder of this subsection. It follows that if $v_{i}$ and $v_{j}$ are vertices of $\Gamma$, then $d\left(v_{i}, v_{j}\right) \in\{0,1,2\}$.

We now record some results which describe the ways in which the connected components of $\Gamma \backslash S_{i}$ and $\Gamma \backslash S_{j}$ may interact for $i \neq j$.

Lemma 4.3. Let $\chi_{i K}, \chi_{j Q} \in \mathcal{P}$. If $d\left(v_{i}, v_{j}\right)=2$ and $v_{j} \notin K$, then $K \cap Q=\emptyset$ or $K \subset Q$.

Proof. Assume that $d\left(v_{i}, v_{j}\right)=2, v_{j} \notin K$ and $K \cap Q \neq \emptyset$. Suppose that $K \not \subset Q$. Let $v_{m} \in K \cap Q$, let $v_{k} \in K \backslash Q$ and let $\alpha$ be a path in $K$ from $v_{m}$ to $v_{k}$. Since $v_{m} \in Q$ but $v_{k} \notin Q$, there exists a vertex $v_{a}$ on $\alpha$ such that $d\left(v_{j}, v_{a}\right)=1$. Since $v_{j}, v_{a} \in \Gamma \backslash S_{i}$ and $d\left(v_{j}, v_{a}\right)=1$, the vertices $v_{a}$ and $v_{j}$ are contained in the same connected component of $\Gamma \backslash S_{i}$. Hence $v_{j} \in K$, contradicting the hypothesis.

Corollary 4.4. Let $\chi_{i K}, \chi_{j Q} \in \mathcal{P}$. Then exactly one of the following nine cases holds:

(1) $d\left(v_{i}, v_{j}\right) \leq 1$

(2) $d\left(v_{i}, v_{j}\right)=2, v_{i} \in Q, v_{j} \in K, K \cap Q=\emptyset$;

(3) $d\left(v_{i}, v_{j}\right)=2, v_{i} \in Q, v_{j} \in K, K \cap Q \neq \emptyset$;

(4) $d\left(v_{i}, v_{j}\right)=2, v_{i} \in Q, v_{j} \notin K, K \cap Q=\emptyset$;

(5) $d\left(v_{i}, v_{j}\right)=2, v_{i} \in Q, v_{j} \notin K, K \subset Q$;

(6) $d\left(v_{i}, v_{j}\right)=2, v_{i} \notin Q, v_{j} \in K, K \cap Q=\emptyset$;

(7) $d\left(v_{i}, v_{j}\right)=2, v_{i} \notin Q, v_{j} \in K, K \supset Q$;

(8) $d\left(v_{i}, v_{j}\right)=2, v_{i} \notin Q, v_{j} \notin K, K \cap Q=\emptyset$;

(9) $d\left(v_{i}, v_{j}\right)=2, v_{i} \notin Q, v_{j} \notin K, K=Q$.

The next two lemmas illustrate the relationship between the connected components of $\Gamma \backslash S_{i}$ and $\Gamma \backslash S_{j}$ and the connected components of $\Gamma \backslash\left(L_{i} \cap L_{j}\right)$. 
Lemma 4.5. Let $1 \leq i<j \leq N$ be such that $d\left(v_{i}, v_{j}\right)=2$ and let $R$ be a subgraph of $\Gamma$. Then $R$ is a connected component of both $\Gamma \backslash S_{i}$ and $\Gamma \backslash S_{j}$ if and only if $R$ is a connected component of $\Gamma \backslash\left(L_{i} \cap L_{j}\right)$ and $v_{i}, v_{j} \notin R$.

Proof. Assume that $R$ is a connected component of both $\Gamma \backslash S_{i}$ and $\Gamma \backslash S_{j}$. Clearly, $v_{i}, v_{j} \notin R$. Since $R$ is a connected subgraph of $\Gamma \backslash S_{i}$ and $L_{i} \cap L_{j} \subset S_{i}, R$ is a connected subgraph of $\Gamma \backslash\left(L_{i} \cap L_{j}\right)$. Suppose that $R$ is not a connected component of $\Gamma \backslash\left(L_{i} \cap L_{j}\right)$. Then there exist $v_{x} \in R, v_{y} \in \Gamma \backslash\left(R \cup\left(L_{i} \cap L_{j}\right)\right)$ such that $d\left(v_{x}, v_{y}\right)=1$. Since $v_{x} \in R$ and $v_{y} \notin R$ and $R$ is a connected component of $\Gamma \backslash S_{i}$, $v_{y} \in S_{i}$. Similarly, $v_{y} \in S_{j}$. Thus $v_{y} \in S_{i} \cap S_{j}=L_{i} \cap L_{j}-$ a contradiction. Hence $R$ is a connected component of $\Gamma \backslash\left(L_{i} \cap L_{j}\right)$.

Now assume that $R$ is a connected component of $\Gamma \backslash\left(L_{i} \cap L_{j}\right)$ and $v_{i}, v_{j} \notin R$. Since $v_{i} \notin R, S_{i} \cap R=\emptyset$ and $R$ is a connected subgraph of $\Gamma \backslash S_{i}$. Suppose that $R$ is not a connected component of $\Gamma \backslash S_{i}$. Then there exist $v_{x} \in R, v_{y} \in \Gamma \backslash\left(R \cup S_{i}\right)$ such that $d\left(v_{x}, v_{y}\right)=1$. Since $v_{x} \in R$ and $v_{y} \notin R$ and $R$ is a connected component of $\Gamma \backslash\left(L_{i} \cap L_{j}\right), v_{y} \in L_{i} \cap L_{j} \subset S_{i}-$ a contradiction. Hence $R$ is a connected component of $\Gamma \backslash S_{i}$. Similarly, $R$ is a connected component of $\Gamma \backslash S_{j}$.

Lemma 4.6. Assume that $\Gamma$ does not contain a SIL. Let $1 \leq i<j \leq N$ be such that $d\left(v_{i}, v_{j}\right)=2$, let $K_{j}$ be the connected component of $\Gamma \backslash S_{i}$ which contains $v_{j}$ and let $Q_{i}$ be the connected component of $\Gamma \backslash S_{j}$ which contains $v_{i}$. Then $\Gamma=K_{j} \cup Q_{i} \cup\left(L_{i} \cap L_{j}\right)$.

Proof. Since $\Gamma$ does not contain a SIL, $\Gamma \backslash\left(L_{i} \cap L_{j}\right)$ has at most two connected components. If $\Gamma \backslash\left(L_{i} \cap L_{j}\right)$ has two connected components, they are $K_{j}$ and $Q_{i}$ and the result is clear. Assume that $\Gamma \backslash\left(L_{i} \cap L_{j}\right)$ is connected. Let $v_{x} \in \Gamma \backslash\left(L_{i} \cap L_{j}\right)$ and let $\alpha$ be a minimal length path in $\Gamma \backslash\left(L_{i} \cap L_{j}\right)$ from $v_{x}$ to $v_{i}$. If $\alpha$ passes through $S_{j}$, then $v_{x} \in K_{j}$. If $\alpha$ does not pass through $S_{j}$, then $v_{x} \in Q_{i}$. Hence the result.

We now consider the algebraic consequences of the graph-theoretic information assembled above. It is convenient to make a list of those cases from Corollary 4.4 in which the relation $\chi_{i K} \chi_{j Q}=\chi_{j Q} \chi_{i K}$ holds and those in which it fails.

Lemma 4.7. We assume the notation of Corollary 4.4. The relation $\chi_{i K} \chi_{j Q}=$ $\chi_{j Q} \chi_{i K}$ holds in cases (1), (5), (7) and (8); it fails in cases (2), (3), (4), (6) and (9).

Proof. In case (1) the hypothesis immediately gives that $v_{i}$ and $v_{j}$ commute; the relation follows immediately. For the remaining cases we determine whether or not the relation holds by computing the images under $\chi_{i K} \chi_{j Q} \chi_{i K}^{-1} \chi_{j Q}^{-1}$ of arbitrary vertices from whichever of the sets $K, Q, K \backslash Q$ and $Q \backslash K$ are appropriate (noting that $\chi_{i K} \chi_{j Q} \chi_{i K}^{-1} \chi_{j Q}^{-1}(v)=v$ for each vertex $v \in \Gamma \backslash(K \cup Q)$ ); if the image of each such vertex is the vertex itself, then the relation holds. We examine two cases in detail, and leave the reader to verify the others using similar methods. 
In case (2) we have $v_{i} \in Q \backslash K, v_{j} \in K \backslash Q$ and we consider an arbitrary element $v_{k} \in K \backslash Q$. Then

$$
\begin{aligned}
v_{k} \stackrel{\chi_{j Q}^{-1}}{\longrightarrow} v_{j}^{-1} v_{k} v_{j} & \stackrel{\chi_{i K}^{-1}}{\longrightarrow} v_{j}^{-1} v_{i}^{-1} v_{k} v_{i} v_{j} \\
& \stackrel{\chi_{j Q}}{\longrightarrow} v_{j}^{-1} v_{i}^{-1} v_{j} v_{k} v_{j}^{-1} v_{i} v_{j} \stackrel{\chi_{i K}}{\longrightarrow} v_{j}^{-1} v_{i}^{-1} v_{j} v_{i} v_{k} v_{i}^{-1} v_{j}^{-1} v_{i} v_{j} .
\end{aligned}
$$

It follows from the hypotheses that $v_{j}^{-1} v_{i}^{-1} v_{j} v_{i}$ is not contained in $W\left(S_{k}\right)$. Lemma 2.4 gives that $v_{j}^{-1} v_{i}^{-1} v_{j} v_{i}$ is not in the centralizer of $v_{k}$. Hence the relation fails.

In case (5) we have $K \subset Q, v_{i} \in Q \backslash K, v_{j} \in \Gamma \backslash(K \cup Q)$ and we consider arbitrary elements $v_{k} \in K$ and $v_{q} \in Q \backslash K$. Then

$$
v_{k} \stackrel{\chi_{j Q}^{-1}}{\longrightarrow} v_{j}^{-1} v_{k} v_{j} \stackrel{\chi_{i K}^{-1}}{\longrightarrow} v_{j}^{-1} v_{i}^{-1} v_{k} v_{i} v_{j} \stackrel{\chi_{j Q}}{\longrightarrow} v_{i}^{-1} v_{k} v_{i} \stackrel{\chi_{i K}}{\longrightarrow} v_{k}
$$

and

$$
v_{q} \stackrel{\chi_{j Q}^{-1}}{\longrightarrow} v_{j}^{-1} v_{q} v_{j} \stackrel{\chi_{i K}^{-1}}{\longrightarrow} v_{j}^{-1} v_{q}^{-1} v_{j} \stackrel{\chi_{j Q}}{\longrightarrow} v_{q} \stackrel{\chi_{i K}}{\longrightarrow} v_{q}
$$

Hence the relation holds.

We are now ready to prove the main result of the section.

Proof of Theorem 1.4. Assume that $W$ is a graph product of directly-indecomposable cyclic groups. By Lemma 4.2, we may assume without loss of generality that there exists a vertex $v \in V_{\Gamma}$ which is adjacent to every other vertex.

Suppose that $\Gamma$ contains a SIL with $i, j$ and $R$ as in Definition 1.3. Let $K_{j}$ denote the connected component of $\Gamma \backslash S_{i}$ which contains $v_{j}$ and let $Q_{i}$ denote the the connected component of $\Gamma \backslash S_{j}$ which contains $v_{i}$. By Lemma 4.5, $\chi_{i R}, \chi_{j R} \in \mathcal{P}$. If $R$ does not contain the least element of $\Gamma \backslash L_{i} \cap L_{j}$, then $\chi_{i R}, \chi_{j R} \in \mathcal{P}^{0}$; the pair $\chi_{i R}, \chi_{j R}$ fits into case (9) of Corollary 4.4, so $\chi_{i R} \chi_{j R} \neq \chi_{j R} \chi_{i R}$ by Lemma 4.7. If $R$ contains the least element of $\Gamma \backslash L_{i} \cap L_{j}$, then $\chi_{i K_{j}}, \chi_{j} Q_{i} \in \mathcal{P}^{0}$; the pair $\chi_{i K_{j}}, \chi_{j} Q_{i}$ fits into case (2) or case (3) of Corollary 4.4, so $\chi_{i K_{j}} \chi_{j} Q_{i} \neq \chi_{j} Q_{i} \chi_{i K_{j}}$ by Lemma 4.7. Hence Out ${ }^{0} W$ is not abelian and property (1) implies property (2).

Now assume that $\Gamma$ does not contain a SIL and let $\chi_{i K}, \chi_{j} Q \in \mathscr{P}^{0}$. By Lemma 4.7, the relation $\chi_{i K} \chi_{j Q}=\chi_{j Q} \chi_{i K}$ holds whenever $d\left(v_{i}, v_{j}\right) \leq 1$. Assume that $d\left(v_{i}, v_{j}\right)=2$. By Lemma 4.6, $\Gamma=K_{j} \cup Q_{i} \cup\left(L_{i} \cap L_{j}\right)$ for $K_{j}$ and $Q_{i}$ as in the statement of the lemma. Without loss of generality, assume that the least element of $\Gamma \backslash\left(L_{i} \cap L_{j}\right)$ is contained in $K_{j}$. By the definition of $\mathcal{P}^{0}, K \neq K_{j}$. Thus $v_{j} \notin K$ and $K \subset Q_{i}$. If $Q=Q_{i}$, then $K \subset Q$ and case (5) of Corollary 4.4 holds. If $Q \neq Q_{i}$, then $v_{i} \notin Q$ and $K \cap Q=\emptyset$ and case (8) of Corollary 4.4 holds. In either case, the relation $\chi_{i K} \chi_{j Q}=\chi_{j Q} \chi_{i K}$ holds by Lemma 4.7. Thus $\operatorname{Out}^{0} W$ is an abelian group and property (2) implies property (1).

Next we assume that $W$ is a graph product of primary cyclic groups. Since each partial conjugation has finite order, it is clear that property (1) implies property 
(3). Suppose that $\Gamma$ contains a SIL with $i, j$ and $R$ as in Definition 1.3. Let $v_{r}$ be a vertex in $R$. Calculation confirms that $\left(\chi_{i R} \chi_{j R}\right)^{n}\left(v_{r}\right)=\left(v_{j} v_{i}\right)^{n} v_{r}\left(v_{j} v_{i}\right)^{-n}$ and $\left(\chi_{i R} \chi_{j R}\right)^{n}\left(v_{i}\right)=v_{i}$ for each positive integer $n$. It follows that no power of $\chi_{i R} \chi_{j R}$ is an inner automorphism. Hence Out $W$, and $\operatorname{Out}^{0} W$, have infinite order and property (3) implies property (2).

4.2. Geometric applications of Theorem 1.4. In this subsection we describe a number of applications of Theorem 1.4.

As mentioned in the introduction, Levitt [23] showed that for a one-ended word hyperbolic group $G$, Out $(G)$ is infinite if and only if $G$ splits over a virtually cyclic subgroup with infinite center, either as an arbitrary HNN extension or as an amalgam of groups with finite center. The following corollary demonstrates that such splittings are not possible in the case that $W$ is a graph product of primary cyclic groups. The proof uses the fact that a graph product of primary cyclic groups is word hyperbolic if and only if every circuit in $\Gamma$ of length four contains a chord [25] and the fact that each separating subgraph of $\Gamma$ corresponds to a splitting of $W$ as a free product with amalgamation (with the separating subgraph generating the amalgamated subgroup).

Corollary 4.8. If $W$ is a graph product of primary cyclic groups and $W$ is a oneended word hyperbolic group, then Out $W$ is finite.

Proof. Let $W$ be a graph product of primary cyclic groups which is one-ended and word hyperbolic. Suppose that $\Gamma$ contains a SIL. By Theorem 1.4, there exist $i, j, R$ as in Definition 1.3. If $L_{i} \cap L_{j}$ is a complete graph, then $W\left(L_{i} \cap L_{j}\right)$ is finite and it follows from the Ends Theorem of Hopf and Stallings (see, for example, [2], Theorem I.8.32) that $W$ has infinitely many ends - a contradiction to the hypothesis. Thus $L_{i} \cap L_{j}$ is not a complete subgraph and there exist non-adjacent vertices $v_{x}, v_{y} \in$ $L_{i} \cap L_{j}$. Then $v_{i} v_{x} v_{j} v_{y}$ is a non-chordal square and $W$ is not word hyperbolic again, a contradiction to the hypothesis.

Remark 4.9. In [27], the authors construct one-ended hyperbolic groups with finite outer automorphism group and a non-trivial JSJ decomposition in the sense of Bowditch (that is, the group has a non-trivial graph of groups decomposition with twoended edge groups and vertex groups which are either two-ended, maximal "hanging Fuchsian", or non-elementary quasiconvex subgroups not of the previous two types - for more details, see [1]). Such groups necessarily have only the trivial JSJ decomposition in the sense of Sela since the outer automorphism groups are finite. Using Corollary 1.4, one may construct examples of right-angled Coxeter groups with similar properties to the groups described in [27]. In particular, if $W$ is a right-angled Coxeter group and the properties

(1) $\Gamma \backslash \Delta$ is connected for each complete subgraph $\Delta$,

(2) every circuit in $\Gamma$ of length four contains a chord, 
(3) $\Gamma \backslash \Lambda$ is disconnected for some subgraph $\Lambda$ which generates a virtually abelian group,

(4) $\Gamma$ has no SIL

hold, then $W$ is a one-ended hyperbolic group with a non-trivial JSJ decomposition in the sense of Bowditch and Out $W$ is finite. For example, the graph $\Gamma$ of Figure 1 has the desired properties (with $\Lambda=\left\{v_{1}, v_{4}\right\}$ ).

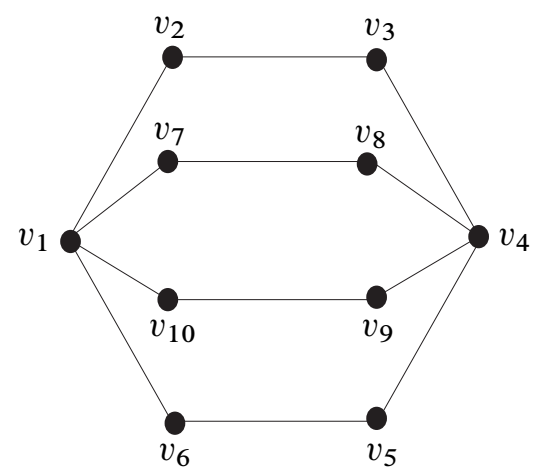

Figure 1. The graph $\Gamma$ for Remark 4.9.

If $W$ is a graph product of primary cyclic groups, then there exists a geometric action of $W$ on a CAT(0) space [25]. We say that $W$ has isolated flats if there exists a geometric action of $W$ on a $\mathrm{CAT}(0)$ space with isolated flats (see [18]). To prove the lemma below we shall need only the following property of such groups, which follows from the results in [18]:

(*) if $W$ has isolated flats and $S_{1}, S_{2} \subseteq W$ are subgroups isomorphic to $\mathbb{Z} \times \mathbb{Z}$ and $S_{1} \cap S_{2} \neq\{\mathrm{id}\}$, then $\left\langle S_{1}, S_{2}\right\rangle$ is virtually abelian.

Lemma 4.10. If $W$ is a graph product of primary cyclic groups and $W$ is one-ended with isolated flats and $1 \leq i<j \leq N$ are such that $d\left(v_{i}, v_{j}\right)=2$, then $W\left(L_{i} \cap L_{j}\right)$ is virtually abelian.

Proof. Let $W, i$ and $j$ be as in the hypothesis of the lemma. Suppose that $W\left(L_{i} \cap L_{j}\right)$ is not virtually abelian. Graph products of primary cyclic groups are subgroups of Coxeter groups [19], Corollary 5.11, and hence linear and satisfy the Tits Alternative. Further, since $W$ acts geometrically on a CAT(0) space, each virtually solvable subgroup is virtually abelian [2], p. 249. It follows that there exist elements $a, b \in W\left(L_{i} \cap L_{j}\right)$ such that $\langle a, b\rangle$ is a free group of rank two. The subgroups $S_{1}=\left\langle v_{i} v_{j}, a\right\rangle$ and $S_{2}=\left\langle v_{i} v_{j}, b\right\rangle$ witness that $W$ does not have property (*), since $\left\langle S_{1} \cup S_{2}\right\rangle$ contains the subgroup $\langle a, b\rangle$. 
Corollary 4.11. If $W$ is a graph product of primary cyclic groups and $W$ is oneended with isolated flats and Out $W$ is infinite, then $W$ splits as a free product with amalgamation $W=A *_{C} B$, where

(1) $A$ and $B$ are special subgroups, and

(2) $C$ is an infinite virtually abelian special subgroup.

Proof of Corollary 4.11. Let $W$ and Out $W$ be as in the hypothesis of the corollary. By Theorem 1.4, there exist $1 \leq i<j \leq N$ such that $d\left(v_{i}, v_{j}\right)=2$ and $L_{i} \cap L_{j}$ separates $\Gamma$. By Lemma 4.10, $W\left(L_{i} \cap L_{j}\right)$ is virtually abelian. Since $W$ is one-ended, $W\left(L_{i} \cap L_{j}\right)$ is not finite. The result follows, with $C=L_{i} \cap L_{j}$.

We say that $W$ has property $(N L C)$ if for every CAT(0) space $X$ on which $W$ acts geometrically, the visual boundary $\partial X$ (see [2], p. 264) is not locally connected.

Corollary 4.12. If $W$ is a right-angled Coxeter group and Out $W$ is infinite, then $W$ has property $(N L C)$.

Proof. Assume that Out $W$ is infinite. By Theorem 1.4, there exist $i, j, R$ as in Definition 1.3. It follows that $W$ is not finite or two-ended. If $W$ has infinitely many ends, then $W$ has property (NLC). Assume that $W$ is one-ended. Since $\left(L_{i} \cap L_{j}, L_{i} \cap L_{j},\left\{v_{i}, v_{j}\right\}\right)$ is a 'virtual factor separator' [26], Definition 3.1, and $L_{i} \cap L_{j}$ is not a 'suspended separator' [26], Definition 3.1, we may apply [26], Theorem 3.2 (2), to conclude that $W$ has property (NLC).

Remark 4.13. We now demonstrate that the converse to Corollary 4.12 does not hold. Let $W$ be the right-angled Coxeter group corresponding to the graph $\Gamma$ in Figure 2. Observe that $\Gamma$ does not contain a SIL, but $\left(\left\{v_{2}, v_{3}, v_{4}\right\},\left\{v_{2}, v_{3}, v_{4}\right\},\left\{v_{1}, v_{6}\right\}\right)$ is a virtual factor separator and $\left\{v_{2}, v_{3}, v_{4}\right\}$ is not a suspended separator. Thus Out $W$ is finite, by Theorem 1.4, and $W$ has property (NLC), by [26], Theorem 3.2 (2).

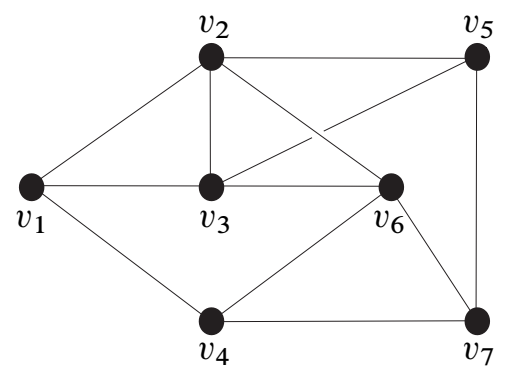

Figure 2. The graph $\Gamma$ for Remark 4.13.

The following application offers a glimpse of some geometry of Aut $W$. 
Corollary 4.14. Let $W$ be a graph product of primary cyclic groups. Then Aut $W$ is word hyperbolic if and only if the following conditions are satisfied:

(1) $\Gamma$ has no SIL;

(2) every circuit in $\Gamma$ of length four contains a chord.

Proof. Assume that $\Gamma$ has an SIL. Let $i, j, R$ be as in Definition 1.3 and let $\iota_{v_{i}} v_{j}$ denote the inner automorphism $w \mapsto v_{i} v_{j} w v_{j}^{-1} v_{i}^{-1}$. Since $d\left(v_{i}, v_{j}\right) \geq 2, \iota_{v_{i}} v_{j}$ has infinite order. The product $\chi_{j R} \chi_{i R}$ also has infinite order and $\left\langle\iota_{v_{i} v_{j}},\left(\chi_{j R} \chi_{i R}\right)\right\rangle \cong \mathbb{Z} \times \mathbb{Z}$. Thus Aut $W$ is not hyperbolic.

Assume that $\Gamma$ has no SIL. By Corollary 1.4 , Out $W$ is finite and Inn $W$ is a finite-index subgroup of Aut $W$. But Inn $W$ is also a finite-index subgroup of $W$ (see Section 2). Thus Aut $W$ and $W$ are commensurable, and hence quasi-isometric (see [2], Example I.8.8.20(1)). The result follows immediately from the characterization of word hyperbolic graph products of primary cyclic groups described above and the fact that word-hyperbolicity is a quasi-isometry invariant [2], Theorem III.H.1.9.

\section{The special case that $\Gamma$ is a tree}

In this section we pursue a more detailed understanding of Out $^{0} W$ in the special case that $\Gamma$ is a tree with at least three vertices. Recall that $L_{i}$ denotes the link of the vertex $v_{i}$. Since $\Gamma$ is a tree, each $W\left(L_{i}\right)$ is a free product of cyclic groups. These subgroups are the building blocks of the direct product decomposition of $\mathrm{Out}^{0} \mathrm{~W}$ in Theorem 1.5. We prove the theorem in Subsection 5.1. We describe some applications of the theorem in Subsection 5.2.

5.1. The proof of Theorem 1.5. For each $1 \leq i \leq N$ we define

$$
\mathscr{L}_{i}^{0}:=\left\{\chi_{j} Q \in \mathcal{P}^{0} \mid d\left(v_{i}, v_{j}\right)=d\left(v_{i}, Q\right)=1\right\} .
$$

So $\chi_{j Q} \in \mathscr{L}_{i}^{0}$ if $v_{i}$ is the unique vertex between $v_{j}$ and $Q$, in which case we say that $v_{i}$ is the link point of $\chi_{j} Q$. Since $\Gamma$ is a tree, each partial conjugation has a unique link point, so the nonempty sets in the list $\mathscr{L}_{1}^{0}, \ldots, \mathscr{L}_{N}^{0}$ form a partition of $\mathcal{P}^{0}$. Note that some of sets $\mathscr{L}_{i}^{0}$ are empty. For example, $\mathscr{L}_{i}^{0}$ is empty if $v_{i}$ is a leaf (that is, adjacent to exactly one vertex).

Lemma 5.1. If $\chi_{i K}, \chi_{j Q} \in \mathscr{P}^{0}$ have distinct link points, then $\chi_{i K}$ and $\chi_{j Q}$ commute.

Proof. We prove the contrapositive. Let $\chi_{i K}, \chi_{j Q} \in \mathcal{P}^{0}$ be elements which do not commute. By Lemma 4.7, one of cases (2), (3), (4), (6) or (9) in Corollary 4.4 must hold. We leave the reader to verify that the definition of $\mathcal{P}^{0}$, combined with the simple geometry of a tree, imply that case (3) is impossible, and cases (2), (4), (6) and (9) may only hold if $\chi_{i K}, \chi_{j} Q$ have a common link point. Thus the result. 
Corollary 5.2. Out $^{0} W=\left\langle\mathscr{L}_{1}^{0}\right\rangle \times\left\langle\mathscr{L}_{2}^{0}\right\rangle \times \cdots \times\left\langle\mathscr{L}_{N}^{0}\right\rangle$ (with some factors trivial).

The following proposition completes the proof of Theorem 1.5. In the statements below, we write $\mathbb{Z}_{\boldsymbol{m}(j)}$ for the cyclic group of order $m(j)$.

Proposition 5.3. Suppose that $\Gamma$ is a tree with at least three vertices. Let $1 \leq i \leq N$ and let $L_{i}=\left\{v_{k_{1}}, v_{k_{2}}, \ldots, v_{k_{M}}\right\}$ with $k_{1}<k_{2}<\cdots<k_{M}$. If $M=1$ (that is, $v_{i}$ is a leaf) or $M>1$ and $v_{k_{2}}$ is the minimal element of $\Gamma \backslash S_{k_{1}}$, then

$$
\left\langle\mathscr{L}_{i}^{0}\right\rangle \cong \mathrm{Out}^{0} W\left(L_{i}\right)
$$

otherwise,

$$
\left\langle\mathscr{L}_{i}^{0}\right\rangle \cong \mathbb{Z}_{\boldsymbol{m}\left(k_{1}\right)} \times \operatorname{Out}^{0} W\left(L_{i}\right) .
$$

Proof. Reindexing the vertices changes the generating set $\mathcal{P}^{0}$, but does not change the isomorphism class of $\mathrm{Out}^{0} W$ (because any two complements of Inn $W$ in $\operatorname{Aut}^{0} W$ are isomorphic). So, by reindexing if necessary, we may assume that indices have been assigned to elements of $V$ so that $v_{1}$ is a leaf and if $d\left(v_{1}, v_{i}\right)<d\left(v_{1}, v_{j}\right)$, then $i<j$.

If $M=1$, then $\mathscr{L}_{i}^{0}=\emptyset$, Out ${ }^{0} W\left(L_{i}\right)$ is trivial and the result holds. So we may assume that $M>1$. Let $\rho_{i}:\left\langle\mathscr{L}_{i}^{0}\right\rangle \rightarrow$ Aut $W\left(L_{i}\right)$ denote the homomorphism determined by restriction, that is, $\left.\chi_{i K} \mapsto \chi_{i K}\right|_{W\left(L_{i}\right)}$.

In this paragraph we show that $\rho_{i}$ is injective. Let $\phi \in \mathscr{L}_{i}^{0}$ be such that $\phi$ acts as the identity on $W\left(L_{i}\right)$. It is immediate from the definition of $\mathscr{L}_{i}^{0}$ that $\phi\left(v_{i}\right)=v_{i}$. We must show that $\phi\left(v_{j}\right)=v_{j}$ for each $j$ such that $v_{j} \in \Gamma \backslash S_{i}$. Fix such an integer $j$. Let $v_{i}=v_{j_{0}}, v_{j_{1}}, \ldots, v_{j_{\ell}}=v_{j} \in V$ be the successive vertices of the unique geodesic from $v_{i}$ to $v_{j}$. So $v_{j_{1}} \in L_{i}$. Let $w_{j_{0}}, w_{j_{1}}, \ldots, w_{j_{\ell}} \in W$ be minimal length elements such that $\phi\left(v_{j_{k}}\right)=w_{j_{k}} v_{j_{k}} w_{j_{k}}^{-1}$. By hypothesis, $w_{j_{0}}=w_{j_{1}}=1$. For each $k=1,2, \ldots, \ell-1$, we have that there exists $u_{k}$ such that $\phi\left(v_{j_{k}}\right)=u_{k} v_{j_{k}} u_{k}^{-1}$ and $\phi\left(v_{j_{k+1}}\right)=u_{k} v_{j_{k+1}} u_{k}^{-1}$. Since $u_{k} v_{j_{k}} u_{k}^{-1}=w_{j_{k}} v_{j_{k}} w_{j_{k}}^{-1}$, we have $w_{j_{k}}^{-1} u_{k}$ is in the centralizer of $v_{j_{k}}$; that is, $w_{j_{k}}^{-1} u_{k} \in\left\langle S_{j_{k}}\right\rangle$. Similarly, $w_{j_{k+1}}^{-1} u_{k} \in\left\langle S_{j_{k+1}}\right\rangle$. So $w_{j_{k+1}}^{-1} w_{j_{k}} \in\left\langle S_{j_{k}} \cup S_{j_{k+1}}\right\rangle$. But $w_{j_{k}}, w_{j_{k+1}} \in W\left(L_{i}\right)$, so $w_{j_{k+1}}^{-1} w_{j_{k}} \in W\left(L_{i}\right) \cap$ $\left\langle S_{j_{k}} \cup S_{j_{k+1}}\right\rangle$. It follows that $w_{j_{k+1}}^{-1} w_{j_{k}} \in\left\langle v_{j_{1}}\right\rangle$ for $k \leq 2$ and $w_{j_{k+1}}^{-1} w_{j_{k}}=1$ for $k \geq 3$. Then it follows that $w_{j_{2}}=1$ and $w_{j_{k}} \in\left\langle v_{j_{1}}\right\rangle$ for each $k \geq 3$. We may assume that $\ell \geq 3$. Let $K_{j}$ denote the connected component of $\Gamma \backslash S_{j_{1}}$ which contains $v_{j}$. Then $K \cap L_{i}=\emptyset$, so $\chi_{j_{1} K} \notin \mathcal{P}_{i}^{0}$. It follows that $v_{j_{1}}$ must have zero exponent sum in $w_{j_{k}}$ for each $3 \leq k \leq \ell$. Hence $w_{j_{k}}=1$ for $3 \leq k \leq \ell$ and, in particular, $\phi\left(v_{j}\right)=v_{j}$.

Finally, we show that the image $\rho_{i}\left(\left\langle\mathscr{L}_{i}^{0}\right\rangle\right)$ is as described in the conclusion of the Proposition. Assume first that the minimal element of $\Gamma \backslash S_{k_{1}}$ is $v_{k_{2}}$. Using the notation $\chi_{k_{j}}\left\{k_{\ell}\right\}:=\chi_{k_{j}\left\{v_{k_{\ell}}\right\}}$, the image $\rho_{i}\left(\mathscr{L}_{i}^{0}\right)$ is as follows:

$$
\rho_{i}\left(\mathscr{L}_{i}^{0}\right)=\left\{\chi_{k_{j}}\left\{k_{\ell}\right\} \mid 1 \leq j, \ell \leq M, j \neq \ell\right\} \backslash\left\{\chi_{k_{1}\left\{k_{2}\right\}}, \chi_{k_{2}\left\{k_{1}\right\}}, \ldots, \chi_{k_{M}\left\{k_{1}\right\}}\right\} .
$$


This is a generating set for $\mathrm{Out}^{0} W\left(L_{i}\right)$. Now assume that $v_{k_{2}}$ is not the minimal element of $\Gamma \backslash S_{k_{1}}$ (so the minimal element of $\Gamma \backslash S_{k_{1}}$ is not contained in $L_{i}$ ). The image $\rho_{i}\left(\mathscr{L}_{i}^{0}\right)$ is as follows:

$$
\rho_{i}\left(\mathscr{L}_{i}^{0}\right)=\left\{\chi_{k_{j}\left\{k_{\ell}\right\}} \mid 1 \leq j, \ell \leq M, j \neq \ell\right\} \backslash\left\{\chi_{k_{2}\left\{k_{1}\right\}}, \ldots, \chi_{k_{M}\left\{k_{1}\right\}}\right\} .
$$

If we replace $\chi_{k_{1}\left\{k_{2}\right\}}$ by the product $\chi_{k_{1}\left\{k_{2}\right\}} \cdots \chi_{k_{1}\left\{k_{M}\right\}}$, then the resulting set still generates $\left\langle\rho_{i}\left(\mathscr{L}_{i}^{0}\right)\right\rangle$. Observe that $\chi_{k_{1}\left\{k_{2}\right\}} \cdots \chi_{k_{1}\left\{k_{M}\right\}}$ commutes with each element in the set $\rho_{i}\left(\mathscr{L}_{i}^{0}\right) \backslash\left\{\chi_{k_{1}\left\{k_{2}\right\}}\right\}$ and $\rho_{i}\left(\mathscr{L}_{i}^{0}\right) \backslash\left\{\chi_{k_{1}\left\{k_{2}\right\}}\right\}$ generates Out ${ }^{0} W\left(L_{i}\right)$. Thus $\rho_{i}\left(\mathscr{L}_{i}^{0}\right)$ generates a subgroup of $\operatorname{Aut}^{0} W\left(L_{i}\right)$ which is isomorphic to $\mathbb{Z}_{\boldsymbol{m}\left(k_{1}\right)} \times$ Out $^{0} W\left(L_{i}\right)$.

Remark 5.4. Our hypotheses on the indexing of $V$ ensure that $v_{1}$ and $v_{N}$ are leaves. One may omit the corresponding terms $\mathrm{Out}^{0} W\left(L_{1}\right)$ and Out ${ }^{0} W\left(L_{N}\right)$ (and any other terms corresponding to leaves) from the statement of Theorem 1.5. However, by including these terms we ensure that the statement stays valid even if the hypotheses on the indexing is dropped.

Remark 5.5. Consider the case that $\Gamma$ is an arbitrary connected graph. Without loss of generality, assume that indices have been assigned to elements of $V$ so that if $d\left(v_{1}, v_{i}\right)<d\left(v_{1}, v_{j}\right)$, then $i<j$. Unlike the tree case, a partial conjugation may have more than one link point and the sets $\mathscr{L}_{i}^{0}$ do not partition $\mathcal{P}^{0}$. However, taking inspiration from the tree case, we define a partition $\mathcal{P}^{0}$ inductively as follows: write $\mathcal{M}_{1}:=\mathcal{P}^{0}$ and for each $1 \leq i \leq N$,

$$
\mathscr{L}_{i}^{\prime}:=\left\{\chi_{j Q} \in \mathcal{M}_{i} \mid v_{i} \text { is a link point of } \chi_{j Q}\right\}, \quad \mathcal{M}_{i+1}:=\mathcal{M}_{i} \backslash \mathscr{L}_{i}^{\prime} .
$$

In some cases, but not all, this partition corresponds to a semi-direct product decomposition of $\mathrm{Out}^{0} W$.

5.2. Applications of Theorem 1.5. We now consider some applications of Theorem 1.5 .

Remark 5.6 (Presenting Aut ${ }^{*} W$ in the case that $\Gamma$ is a tree). Since Ab is the direct product $\prod_{k \in K} \mathbb{Z}_{\boldsymbol{m}(k)}$, where $K$ is the set

$\left\{k \mid\right.$ there exists $i$ such that $1 \leq i, k \leq N, v_{k}$ is the minimal vertex in $L_{i}$ and $L_{i}$ does not contain the minimal vertex of $\left.\Gamma \backslash S_{k}\right\}$,

one may write down a finite presentation of Ab. Since each $W\left(L_{i}\right)$ is a free product of cyclic groups, one may use work of Fouxe-Rabinovitch [11] (see also [24], footnote 1, p. 1) and Gilbert [12] to write down a finite presentation for $\mathrm{Out}^{0} W\left(L_{i}\right)$. Combining these presentations in the standard way for presenting a direct product gives a finite presentation for $\mathrm{Out}^{0} W$. Further, a finite presentation for Inn $W$ is well-known 
(cf. Lemma 2.7) and, because each maximal complete subgroup is a direct product of two cyclic groups, it is an easy exercise to write down a finite presentation of $\mathrm{Aut}^{1} W$. Combining the presentations of Inn $W$, Out ${ }^{0} W$ and Aut $^{1} W$ in the standard way for presenting semi-direct products (including computing the image of each generator of the normal factor under conjugation by each generator of the other factor) one is then able to write down a finite presentation of Aut $^{*} W$ (cf. [21], [28]).

Recall that we write $V$ (resp. E) for the set of vertices (resp. edges) of $\Gamma$ and $N=|V|$. Let $V_{1} \subset V$ denote the set of vertices which have valence one (the 'leaves' of $\Gamma)$.

Corollary 5.7. If $W$ is a graph product of primary cyclic groups and $\Gamma$ is a tree, then Out $W$ is virtually torsion-free and

$$
\operatorname{vcd}(\text { Out } W)=\left|V_{1}\right|-2 .
$$

Proof. First it follows from Lemma 2.8(1) and Theorem 1.5 that the product $\prod_{i=1}^{N} \operatorname{Out}^{0} W\left(L_{i}\right)$ is isomorphic to a subgroup of finite index in Out $W$. Thus it suffices to calculate the virtual cohomological dimension of this product.

For each $i, W\left(L_{i}\right)$ is a free product of finite groups and so $\mathrm{Out}^{0} W\left(L_{i}\right)$ is virtually torsion-free [8] and $\operatorname{vcd}\left(\mathrm{Out}^{0} W\left(L_{i}\right)\right)=\max \left\{0,\left|L_{i}\right|-2\right\}$ [20] [24], p. 67. The direct product of virtually torsion-free groups is virtually torsion-free, so $\prod_{i=1}^{N} \mathrm{Out}^{0} W\left(L_{i}\right)$ is virtually torsion-free.

It follows from [3], Proposition VII.2.4 (b), p. 187 (see also [3], VIII.11, Exercise 2, p. 229) that the virtual cohomological dimension of a direct product is at most the sum of the virtual cohomological dimensions of the factors. Thus we have

$$
\operatorname{vcd}\left(\prod_{i=1}^{N} \operatorname{Out}^{0} W\left(L_{i}\right)\right) \leq \sum_{i=1}^{N} \max \left\{0,\left|L_{i}\right|-2\right\} .
$$

For each $1 \leq i \leq N$, Out $^{0} W\left(L_{i}\right)$ contains a free abelian subgroup of rank $\max \left\{0,\left|L_{i}\right|-2\right\}$ (if the subgraph $L_{i}=\left\{v_{j_{1}}, \ldots, v_{j_{M}}\right\}$, then $\left\{\left(\chi_{j_{2}\left\{j_{3}\right\}} \chi_{j_{1}\left\{j_{3}\right\}}\right), \ldots\right.$, $\left.\left(\chi_{j_{2}\left\{j_{M}\right\}} \chi_{j_{1}\left\{j_{M}\right\}}\right)\right\}$ generates a free abelian subgroup). It follows that the product $\prod_{i=1}^{N} \operatorname{Out}^{0} W\left(L_{i}\right)$ contains a free abelian subgroup of rank

$$
\sum_{i=1}^{N} \max \left\{0,\left|L_{i}\right|-2\right\}
$$

and

$$
\operatorname{vcd}\left(\prod_{i=1}^{N} \operatorname{Out}^{0} W\left(L_{i}\right)\right)=\sum_{i=1}^{N} \max \left\{0,\left|L_{i}\right|-2\right\} .
$$


Finally,

$$
\begin{aligned}
\sum_{i=1}^{N} \max \left\{0,\left|L_{i}\right|-2\right\} & =\left(\sum_{i=1}^{N}\left(\left|L_{i}\right|-2\right)\right)+\left|V_{1}\right| \\
& =\left(\sum_{i=1}^{N}\left|L_{i}\right|\right)-2 N+\left|V_{1}\right| \\
& =2|E|-2|V|+\left|V_{1}\right| \\
& =2|E|-2(|E|+1)+\left|V_{1}\right| \\
& =\left|V_{1}\right|-2 .
\end{aligned}
$$

(the first equality holds because $\left|L_{i}\right|-2<0$ if and only if $v_{i} \in V_{1}$ and $\left|L_{i}\right|-2=-1$, the third equality holds because each edge in $\Gamma$ contributes to $\left|L_{i}\right|$ for two values of $i$ and the fourth equality holds because $|V|=|E|+1$ ).

The following corollary extends the main results from [15].

Corollary 5.8. If $W$ is a right-angled Artin group and $\Gamma$ is a tree, then there exist regular languages of normal forms for $\mathrm{Out}^{0} W$ and $\mathrm{Aut}^{0} W$.

Proof. Consider the structure of $\mathrm{Out}^{0} \mathrm{~W}$ as described in Theorem 1.5. For each $1 \leq i \leq N, W\left(L_{i}\right)$ is a free group and there exists a regular language of normal forms $\mathcal{N}_{i}$ for $\mathrm{Out}^{0} W\left(L_{i}\right)$ [15]. Since $\mathrm{Ab}$ is a finitely generated free abelian group, there is a regular language of normal forms $\mathcal{N}_{\mathrm{Ab}}$ for Ab. The language $\mathcal{N}_{\mathrm{Ab}} \mathcal{N}_{1} \mathcal{N}_{2} \ldots \mathcal{N}_{N}$ is a regular language of normal forms for $\mathrm{Out}^{0} W$.

Further, Inn $W$ is a right-angled Artin group and hence is automatic [17], Theorem B. It follows that there is a regular language of normal forms $\mathcal{N}_{I}$ for Inn $W$. By Theorem 3.2, the language $\mathcal{N}_{I} \mathcal{N}_{\mathrm{Ab}} \mathcal{N}_{1} \mathcal{N}_{2} \ldots \mathcal{N}_{N}$ is a regular language of normal forms for $\mathrm{Aut}^{0} \mathrm{~W}$.

\section{References}

[1] B. H. Bowditch, Cut points and canonical splittings of hyperbolic groups. Acta Math. 180 (1998), 145-186. Zbl 0911.57001 MR 1638764

[2] M. R. Bridson and A. Haefliger, Metric spaces of non-positive curvature. Grundlehren Math. Wiss. 319, Springer-Verlag, Berlin 1999. Zbl 0988.53001 MR 1744486

[3] K. S. Brown, Cohomology of groups. Graduate Texts in Math. 87, Springer-Verlag, New York 1982. Zbl 0584.20036 MR 0672956

[4] K.-U. Bux, R. Charney, and K. Vogtmann, Automorphisms of two-dimensional RAAGS and partially symmetric automorphisms of free groups. Groups Geom. Dyn. 3 (2009), 541-554. Zbl 05651408 MR 2529947

[5] A. Castella, Sur les automorphismes et la rigidité des groupes de Coxeter à angles droits. J. Algebra 301 (2006), 642-669. Zbl 1104.20037 MR 2236762 
[6] R. Charney, J. Crisp, and K. Vogtmann, Automorphisms of 2-dimensional right-angled Artin groups. Geom. Topol. 11 (2007), 2227-2264. Zbl 1152.20032 MR 2372847

[7] R. Charney and K. Vogtmann, Finiteness properties of automorphism groups of right-angled Artin groups. Bull. Lond. Math. Soc. 41 (2009), 94-102. Zbl 05530801 MR 2481994

[8] D. J. Collins, The automorphism group of a free product of finite groups. Arch. Math. (Basel) 50 (1988), 385-390. Zbl 0654.20036 MR 942533

[9] D. J. Collins and N. D. Gilbert, Structure and torsion in automorphism groups of free products. Quart. J. Math. Oxford Ser. (2) 41 (1990), 155-178. Zbl 0693.20039 MR 1053660

[10] M. B. Day, Peak reduction and finite presentations for automorphism groups of rightangled Artin groups. Geom. Topol. 13 (2009), 817-855. Zbl 1226.20024 MR 2470964

[11] D. I. Fouxe-Rabinovitch, Über die Automorphismengruppen der freien Produkte. I. Rec. Math. [Mat. Sbornik] N.S. 8(50) (1940), 265-276. JFM 66.0066.04 Zbl 0025.00903 MR 0003413

[12] N. D. Gilbert, Presentations of the automorphism group of a free product. Proc. London Math. Soc. (3) 54 (1987), 115-140. Zbl 0609.20023 MR 872253

[13] E. R. Green, Graph products of groups. PhD thesis, The University of Leeds, Leeds 1990. http://etheses.whiterose.ac.uk/236/

[14] M. Gutierrez and A. Kaul, Automorphisms of right-angled Coxeter groups. Int. J. Math. Math. Sci. 2008 (2008), Art. ID 976390. Zbl 1152.20035 MR 2439005

[15] M. Gutiérrez and S. Krstić, Normal forms for basis-conjugating automorphisms of a free group. Internat. J. Algebra Comput. 8 (1998), 631-669. Zbl 0960.20021 MR 1682236

[16] M. Gutierrez and A. Piggott, Rigidity of graph products of abelian groups. Bull. Aust. Math. Soc. 77 (2008), 187-196. Zbl 1173.20018 MR 2428781

[17] S. Hermiller and J. Meier, Algorithms and geometry for graph products of groups. $J$. Algebra 171 (1995), 230-257. Zbl 0831.20032 MR 1314099

[18] G. C. Hruska and B. Kleiner, Hadamard spaces with isolated flats. Geom. Topol. 9 (2005), 1501-1538; erratum to "Hadamard spaces with isolated flats", ibid. 13 (2009), 699-707. Zbl 1087.20034 MR 2175151 Zbl 1176.20046 MR 2469527

[19] T. Januszkiewicz and J. Świątkowski, Commensurability of graph products. Algebr. Geom. Topol. 1 (2001), 587-603. Zbl 0998.20029 MR 1875609

[20] S. Krstić and K. Vogtmann, Equivariant outer space and automorphisms of free-by-finite groups. Comment. Math. Helv. 68 (1993), 216-262. Zbl 0805.20030 MR 1214230

[21] M. R. Laurence, Automorphisms of graph products of groups. PhD thesis, Queen Mary College, University of London, London 1993.

[22] M. R. Laurence, A generating set for the automorphism group of a graph group. J. London Math. Soc. (2) 52 (1995), 318-334. Zbl 0836.20036 MR 1356145

[23] G. Levitt, Automorphisms of hyperbolic groups and graphs of groups. Geom. Dedicata 114 (2005), 49-70. Zbl 1107.20030 MR 2174093

[24] D. McCullough and A. Miller, Symmetric automorphisms of free products. Mem. Amer. Math. Soc. 122 (1996), no. 582. Zbl 0860.20029 MR 1329943 
[25] J. Meier, When is the graph product of hyperbolic groups hyperbolic? Geom. Dedicata 61 (1996), 29-41. Zbl 0874.20026 MR 1389635

[26] M. Mihalik, K. Ruane, and S. Tschantz, Local connectivity of right-angled Coxeter group boundaries. J. Group Theory 10 (2007), 531-560. Zbl 1184.20040 MR 2334758

[27] C. F. Miller, III, W. D. Neumann, and G. A. Swarup, Some examples of hyperbolic groups. In Geometric group theory down under (Canberra, 1996), Walter de Gruyter, Berlin 1999, 195-202. Zbl 0955.20027 MR 1714846

[28] B. Mühlherr, Automorphisms of graph-universal Coxeter groups. J. Algebra 200 (1998), 629-649. Zbl 0897.20033 MR 1610676

[29] H. Servatius, Automorphisms of graph groups. J. Algebra 126 (1989), 34-60. Zbl 0682.20022 MR 1023285

[30] J. Tits, Sur le group des automorphismes de certains groupes de Coxeter. J. Algebra 113 (1988), 346-357. Zbl 0657.20027 MR 929765

Received August 17, 2007; revised May 25, 2010

M. Gutierrez, Department of Mathematics, Tufts University, Medford, MA 02155, U.S.A.

E-mail: Mauricio.Gutierrez@tufts.edu

A. Piggott, Department of Mathematics, Bucknell University, Lewisburg, PA 17837, U.S.A.

E-mail: adam.piggott@bucknell.edu

K. Ruane, Department of Mathematics, Tufts University, Medford, MA 02155, U.S.A.

E-mail: Kim.Ruane@tufts.edu 